\title{
Aqueous Extract of Cimicifuga dahurica Reprogramming Macrophage Polarization by Activating TLR4-NF-kB Signaling Pathway
}

\author{
Shushu Qian $\mathbb{D}^{1}{ }^{\prime} *$, Xuan Han ${ }^{2, *}$, Xiaocao Sha',*, Fang Tian', Hong Huang', Pengjun Jiang', \\ Guoshun Huang', Bangyun Ma', Hong Zhang', Yiye Zhu', Xuemei Sun' \\ 'Jiangsu Province Hospital of Chinese Medicine, Affiliated Hospital of Nanjing University of Chinese Medicine, Nanjing, Jiangsu, People's Republic of \\ China; ${ }^{2}$ School of Integrated Chinese and Western Medicine, Nanjing University of Chinese Medicine, Nanjing, Jiangsu, People's Republic of China \\ *These authors contributed equally to this work \\ Correspondence: Xuemei Sun, Tel +86-25-866I7I4I, Fax+86-25-86518690, Email 139139II666@qq.com
}

\begin{abstract}
Purpose: Cimicifuga dahurica (C. dahurica), which has been used in traditional oriental medicine for a long period, was reported to exert extensive antitumor activity, but the effect and molecular biological mechanism of $C$. dahurica on multiple myeloma (MM) has not been elaborated. Tumor-associated macrophages (TAMs) exhibit a sustained polarization between tumor killing M1 subtype and tumor supporting M2 subtype. And a lower ratio of M1/M2 is associated with tumor angiogenesis, proliferation and invasion. We explored the inhibitory effect of the aqueous extract of the root of C. dahurica (CRAE) on tumor growth by reprogramming macrophage polarization in the tumor microenvironment.
\end{abstract}

Methods: Mice bearing SP2/0 multiple myeloma were treated with CRAE. Western blotting (WB), immunohistochemistry (IHC) and immunofluorescence staining were utilized to assess tumor growth and TAM populations. Macrophages were depleted by injection of clodronate liposomes to determine and measure the role of CRAE as an anti-tumor agent by targeting macrophages. To simulate tumor microenvironment, MM cells H929 and TAMs were co-cultured using the transwell co-culture system. By using CRAE as an immunoregulator in M2-like macrophages, we analyzed CRAE-treated macrophage-associated surface markers and cytokines by flow cytometry and WB.

Results: The results indicated that CRAE treatment could reduce tumor burden of MM mice and a high degree of M1-like macrophages infiltration was detected in tumor tissues. In vitro co-culture system, CRAE significantly promoted the polarization of M2 to M1 phenotype, which led to the increase in apoptosis of myeloma cells. It was found that the M1 polarization induced by CRAE depended on the TLR4-MyD88-TAK1-NF-KB signal transduction.

Conclusion: This study elucidated the anticancer mechanism of the aqueous extract of $C$. dahurica (CRAE) through reprogramming macrophage polarization and highlighted that CRAE could act as a potential novel option for cancer immunotherapy.

Keywords: Cimicifuga dahurica, tumor-associated macrophages, macrophage polarization, multiple myeloma, tumor microenvironment

\section{Introduction}

Malignant proliferation of monoclonal plasma cells in bone marrow is a pathological feature of multiple myeloma (MM). As a malignant hematologic disease, the incidence rate of MM is second only to lymphoma. ${ }^{1}$ It usually results in end organ damage, such as anemia, renal dysfunction, osteolytic lesions and hypercalcemia, which seriously threatens the life and health of patients. Although the prognosis of patients has been significantly improved in the era of targeting myeloma cells and immunomodulator treatment, it still remains largely incurable and will eventually relapse and develop refractory disease. $^{2}$ Accumulating evidence suggests that tumor microenvironment plays a vital role in tumor progression. The interaction of malignant plasma cells and the bone marrow constituents promotes tumor growth, proliferation, invasion, 
metastasis and drug resistance. ${ }^{3}$ Tumor-associated macrophages (TAMs) are abundantly present in the MM microenvironment. Activated macrophages have two polarization states: classically activated M1-like macrophages and alternatively activated M2-like macrophages subtypes. M1-like macrophages are activated by Interferon- $\gamma$ (IFN- $\gamma$ ), tumor necrosis factor- $\alpha$ (TNF- $\alpha$ ), or bacterial lipopolysaccharide (LPS), participating in antigen presentation and exerting cytotoxic effects on tumor cells, while M2 induced by interleukin-4 (IL-4), interleukin-10 (IL-10) and interleukin-13 (IL-13) could support tumor cell proliferation, enhance angiogenesis and form an immunosuppressive environment. ${ }^{4}$ Studies have shown that TAMs are composed of M1-like and M2-like subtypes, which are in dynamic change. With the tumor progression, TAMs gradually adopt to M2-like phenotype. Recent studies have shown that the degree of TAM infiltration is correlated with the poor prognosis of MM, which can be used as a potential index of prognosis. ${ }^{5,6} \mathrm{MM}$ associated macrophages promote angiogenesis through vasculogenic mimicry and protect MM cells from spontaneous and chemotherapy-induced apoptosis via contact-dependent and contact-independent mechanisms. ${ }^{7,8}$ Therefore, MMassociated macrophages have become a potential target for the treatment of myeloma. ${ }^{9,10}$

Natural products remain a major resource for modern drug development and exert an important role in comprehensive tumor treatment. Finding and developing natural plant products with high efficiency and low toxicity is one of the directions of MM-associated clinical drug research and development. Shengma Biejia decoction (SMBJD), a classic prescription of Traditional Chinese Medicine (TCM) for treatment of autoimmune diseases and hemorrhagic diseases, has been used for several thousand years in China. It comes from the Golden Chamber (also called JinGuiYaoLue) written by Zhongjing Zhang. Previous clinical studies showed that the treatment of MM with SMBJD combined with Thalidomide metronomic chemotherapy had obvious therapeutic effect. ${ }^{11}$ SMBJD can significantly inhibit the growth of myeloma in nude mice and significantly improve leukocytes, erythrocytes and hemoglobin except platelets. ${ }^{12}$ Cimicifugae rhizoma (Shengma) is the main element in SMBJD. The genus Cimicifuga (now known as Actaea), belonging to the family Ranunculaceae, consists of 28 species worldwide. ${ }^{13}$ Cimicifugae rhizome which encompasses three Cimicifuga species, namely, Cimicifuga dahurica (Turcz.) Maxim. (C. dahurica), Cimicifuga heracleifolia Kom. and Cimicifuga foetida L. is commonly known in Chinese medicine as "Shengma", officially listed in the Chinese Pharmacopoeia. In traditional Chinese medicine, Cimicifugae rhizoma has been extensively used for treating sore throat, toothache, wind-heat headache, uterine prolapse, archoptosis and other diseases. ${ }^{13,14} \mathrm{Up}$ to now, more than 200 compounds including triterpenoids and their glycosides, phenolic acids and chromogen ketones have been isolated. ${ }^{13}$ Domestic and foreign scholars have carried out systematic research on C. dahurica and found that $C$. dahurica showed advanced antitumor activity in vivo and in vitro, but the effect of $C$. dahurica on MM and its molecular biological mechanism have not been clarified.

Cimicifugae rhizome and the decoction with Cimicifugae rhizome as the main component have been used in the treatment of MM in China for a long time, known as playing an important role in prolonging the life of cancer patients and improving their quality of life. However, its anti-myeloma mechanism remains to be found. ${ }^{13,14}$ Collectively, we selected the aqueous extract of the root of $C$. dahurica (CRAE) as the research object in this study, based on the characteristic theory of TCM. And we deeply excavated the material basis and related mechanism of C. dahurica targeting TAMs in order to make $C$. dahurica used in clinic more scientifically and widely. This work may advance our understanding of the anti-myeloma effect of $C$. dahurica.

\section{Materials and Methods}

\section{Reagents and Antibodies}

Roswell Park Memorial Institute RPMI 1640 medium (RPMI 1640) (cat\#15630-056) and fetal bovine serum (FBS) (cat\#10099141C) were purchased from Invitrogen (Carlsbad, CA, USA). RIPA buffer (cat\#P0013B), phosphatase and protease inhibitor cocktail (cat\#P1048), TUNEL apoptosis assay kit (cat\#C1090) and BCA Protein Assay Kit (cat\#P0011) were purchased from Beyotime (Shanghai, China). Annexin V-FITC/PI apoptosis kit (cat\#AP101-100-kit) was purchased from MultiSciences (Hangzhou, China). Phorbol 12-myristate 13-acetate (PMA) (cat\#FMS-FZ207) was purchased from FcMACS (Nanjing, China). Recombinant human IL-13 (cat\#213-ILB-025), recombinant human IFN- $\gamma$ 
(cat\#285-IF-100) and recombinant human IL-4 (cat\#204-IL-020) were purchased from R\&D (MN, USA). Lipopolysaccharide (cat\#L2630) was purchased from Sigma-Aldrich (MO, USA).

For Western blotting, anti-Arginase 1 (cat\#93668), anti-iNOS (cat\#20609), anti-beta actin (cat\#3700), anti-CD163 (cat\#93498) and anti-p65 (cat\#8242) were purchased from CST (MN, USA). Anti-CD86 (cat\#AF300280) was purchased from AiFang (Hunan, China). Anti-cleaved-IL-1beta (cat\#AF4006) was purchased from Affinity Biosciences (OH.USA). For flow cytometry, PE anti-human CD163 (cat\#326506) and Alexa Fluor 488 anti-human CD86 (cat\#305414) were purchased from BioLegend (CA, USA). Bortezomib (cat\#HY-10227), TAK-242 (cat\#HY-11109) and BAY 11-7085 (cat\#HY-10257) were purchased from MedChemExpress (NJ, USA). For the preparation and polarization of bone marrow-derived macrophages (BMDMs), red blood cell (RBC) lysis buffer (cat\# 00-4333-57) was purchased from Thermo Fisher Scientific (MA, USA). Mouse macrophage colony-stimulating factor (M-CSF) (cat\#416-ML-050), mouse IL-4 (cat\#404-ML-050) and IL-13 (cat\#413-ML-050) were purchased from R\&D Systems (MN, USA). For macrophage depletion, Clophosome ${ }^{\circledR}$-clodronate liposomes (CL) (cat\#40337ES10) and negative control PBS liposome (PL) (cat\#40338ES08) were purchased from Yeasen (Shanghai, China). For immunohistochemistry staining and immunofluorescence staining, anti-CD163 (cat\# GB14027), anti-iNOS (cat\#GB11119), anti-Arginase-1 (cat\#GB11285), antiCD86 (cat\#GB13585) and anti-F4/80 (cat\#GB11027) were purchased from Servicebio (Wuhan, China).

\section{Preparation of the Aqueous Extract of the Root of $C$. dahurica (CRAE)}

C. dahurica was sourced from Jiangsu Province Hospital of TCM (Nanjing, China) and was appraised by a Chinese pharmacist. The preparation method of the aqueous extract of the root of $C$. dahurica is as follows: first, we soaked the root of $C$. dahurica $(100 \mathrm{~g})$ in double-distilled water with volume of $1000 \mathrm{~mL}$ for $30 \mathrm{~min}$, then we boiled them at $180{ }^{\circ} \mathrm{C}$ for $30 \mathrm{~min}$ to collect the extract. And the materials were boiled again in $1000 \mathrm{~mL}$ of double-distilled water with $120^{\circ} \mathrm{C}$ for $60 \mathrm{~min}$, then we mixed the two extracts, boiled and evaporated them to $100 \mathrm{~mL}$. The final stock solution of the extract was determined to be $1 \mathrm{~g} / \mathrm{mL}$. We filtered the extract through a $0.22 \mu \mathrm{m}$ filter and stored it at $-20{ }^{\circ} \mathrm{C}$. The levels of endotoxin were assessed by limulus amebocyte lysate (LAL) assay purchased from GenScript (NJ, USA). Specifically, we diluted the sample at 1:1000, adjusted the $\mathrm{pH}$ value, and incubated it in LAL for $10 \mathrm{~min}$ at $37^{\circ} \mathrm{C}$. After adding the chromogenic substrate, the absorbance was read at $405 \mathrm{~nm}$ and compared with E. coli standard. The endotoxin in the final grade was less than $0.1(\mathrm{EU}) / \mathrm{mL}$.

\section{UHPLC-ESI- Q-Orbitrap-MS/MS Analysis of CRAE}

UHPLC-ESI- Q-Orbitrap-MS/MS analysis was conducted with a Thermo Fisher UltiMate 3000 RS ultra-high performance liquid chromatography (UHPLC). Separation was performed using an UHPLC column (RP-C18 2.1×150 mm, 1.8 $\mu \mathrm{m})$ operated at $35{ }^{\circ} \mathrm{C}$. Mobile phases were $0.1 \%(\mathrm{v} / \mathrm{v})$ formic aqueous solution (A) and $0.1 \%(\mathrm{v} / \mathrm{v})$ formic acid acetonitrile (B). The flow rate was $0.30 \mathrm{~mL} / \mathrm{min}$ and gradient elution was as follows: $0-1 \mathrm{~min}, 2 \% \mathrm{~B} ; 1-5 \mathrm{~min}, 2-$ $20 \%$ B; 5-10 min, 20-50\% B; 10-15 min, 50-80\% B; 15-20 min, 80-95\% B; 20-25 min, 95\% B; 25-26 min, 2-95\% B; 26-30 min, 2\% B. The MS parameters were optimized as follows: sheath gas flow rate (40 arb), aux gas and heater temp (nitrogen, $350{ }^{\circ} \mathrm{C}$ ), capillary temperature $\left(300{ }^{\circ} \mathrm{C}\right)$, positive spray voltage $(3.8 \mathrm{kv})$. Positive and negative full scan data were obtained at a resolving power of 70,000 FWHM. A scan range of m/z 150.0-2000.0 was set for the MS analysis.

\section{Animals}

We purchased 40 female BALB/c nude mice from the Jiangsu Jicui Yaokang Biotechnology Co., Ltd. The average age of mice was 4-6 weeks, and the average body weight was $20 \mathrm{~g}$. The mice were housed in a clean environment and had access to standard food and water according to the "guidelines of animal use and care". After 7 days of acclimatization, 8 mice were stochastically conducted as the blank group and the other 32 mice were conducted as the experimental groups. SP2/0 cells at the logarithmic growth stage were collected and the adjusted concentration $\left(2.5 \times 10^{6}\right.$ cells $)$ were injected into the left forelimb of each experimental mice after the cell density was adjusted to $2.5 \times 10^{6}$ cells $/ \mathrm{mL}$. On day 8 after inoculation, the tumor volume of the mice reached about $80-100 \mathrm{~mm}^{3}$, and the mice were randomly divided into four groups (eight mice each group): Vehicle group (double-distilled water, $0.2 \mathrm{~mL} / \mathrm{mouse}$, gavage, once a day, NS, $0.1 \mathrm{~mL} /$ mouse, intraperitoneal injection, twice a week); CRAE group (CRAE $78 \mathrm{mg} / 0.2 \mathrm{~mL} / \mathrm{mouse}$, gavage, once a day; NS 
$0.1 \mathrm{~mL} / \mathrm{mouse}$, intraperitoneal injection, twice a week); Bortezomib group (double-distilled water, $0.2 \mathrm{~mL} / \mathrm{mouse}$, gavage, once a day and bortezomib $6.01674 \mu \mathrm{g} / 100 \mu \mathrm{L} /$ mouse, intraperitoneal injection, twice a week); Combo group (CRAE $78 \mathrm{mg} / 0.2 \mathrm{~mL} /$ mouse via gavage and bortezomib $6.01674 \mu \mathrm{g} / 100 \mu \mathrm{L} / \mathrm{mouse}$ via intraperitoneal injection, twice a week). The length and width of the tumor were measured every 3 days, and the tumor volume was calculated by $\frac{\text { length } \times \text { width }}{2}$. Meanwhile, the psychological state of mice was recorded. On the 45 th day, the mice were euthanized by cervical posterior dislocation after anesthesia, and the tumors were harvested and weighed. Liver, spleen and kidney were dissected from mice. The Experimental Animal Ethics Committee of Nanjing University of Chinese Medicine approved the development of this animal experiment. All animal experiments conformed to the Guidelines for the Care and Use of Laboratory Animals and were approved by the Animal Ethics Committee of the Affiliated Hospital of Nanjing University of Traditional Chinese Medicine (permit number: 2021DW-28-02).

\section{Mouse Bone Marrow-Derived Macrophages (BMDMs) Preparation and Polarization}

The femurs of C57BL/6 mice ( $8-10$ weeks old) were rinsed with pre-cooled $1 \times \mathrm{PBS}$ and bone marrow was collected. After collection, red blood cells (RBC) lysis buffer were used to lyse RBC, and the remaining cells were washed twice with $1 \times$ PBS. Sorted bone-marrow cells or monocytes were cultured in RPMI 1640 medium containing $10 \%$ fetal bovine serum and $20 \mathrm{ng} / \mathrm{mL}$ M-CSF to induce differentiation of macrophages. We replaced the medium with fresh medium containing M-CSF every 3 days. From day 7, M2-like polarization was achieved by using mouse $20 \mathrm{ng} / \mathrm{mL}$ IL-4 and 20 $\mathrm{ng} / \mathrm{mL}$ IL-13 for 2 consecutive days. After polarization, phenotypes of cells were identified and used for different assays. Culture medium from M2 macrophages and CRAE-treated M2 macrophages were collected for flow cytometry and ELISA assays.

\section{Macrophage Depletion}

Clodronate liposomes (CL) and negative control PBS liposomes (PL) were removed from the refrigerator and restored to room temperature $\left(18^{\circ} \mathrm{C}\right)$. CL and PL were turned upside down for 7-9 times and mixed well and then $200 \mu \mathrm{L} C L(1 \mathrm{mg})$ or $200 \mu \mathrm{L}$ PL were injected intraperitoneally per $20 \mathrm{~g}$ of body weight of per mouse. Intraperitoneal injections were repeated in animal models twice a week for 2 weeks to deplete macrophage.

\section{Hematoxylin and Eosin Staining}

Fresh tissues that had been fixed in $4 \%$ paraformaldehyde solution for at least 24 hours were embedded in paraffin, and the paraffin blocks were cut into $4 \mu \mathrm{m}$ paraffin sections. Paraffin sections were dewaxed with xylene, stained in Harris hematoxylin solution for 3-8 $\mathrm{min}$, rinsed with distilled water for $5 \mathrm{~min}$, differentiated with $1 \%$ hydrochloric acid alcohol for $20 \mathrm{~s}$ and rinsed again with distilled water. After turned blue with $0.6 \%$ ammonia, the sections were washed with tap water, and then stained with $0.5 \%$ eosin for 1-3 minutes. Finally, the sections were soaked in different concentrations of alcohol gradient dehydration and could be sealed with neutral resin after xylene penetration and drying. All the above reagents were purchased from Servicebio Biotechnology Co., Ltd. (Wuhan, China). Slides were scanned using a Pannoramic 250 Flash III (3dhistech, Budapest, Hungary).

\section{Immunohistochemistry Staining}

Paraformaldehyde-fixed tumor tissues fixed with paraformaldehyde were embedded in paraffin and cut into 4-mm-thick sections, dewaxed, and hydrated. The sections were boiled for $5 \mathrm{~min}$ in $10 \mathrm{mM}$ sodium citrate buffer and cooled at room temperature for $30 \mathrm{~min}$, followed by being incubated at 3\% hydrogen peroxide for $10 \mathrm{~min}$ to reduce endogenous peroxidase activity. Then, after washed with $1 \times \mathrm{PBS}$ for 3 times, the sections were blocked using goat serum for $30 \mathrm{~min}$. Next, the sections were incubated with the primary antibody anti-CD163, anti-iNOS, anti-Arginase-1 and anti-CD86 for $2 \mathrm{~h}$ at $37^{\circ} \mathrm{C}$. After the antibody was removed, the sections were cleaned three times again with PBS. Then, anti-rabbit or anti-mouse IgG were used to incubate the sections (Servicebio, Wuhan, China) for $30 \mathrm{~min}$ at $37{ }^{\circ} \mathrm{C}$. The sections were washed again with PBS for 3 times. Finally, diaminobenzidine solution was used for color rendering, and hematoxylin was used to stain. 


\section{Immunofluorescence Staining}

Anti-F4/80 (1:200) and anti-CD86 (1:200) were used to label cells to calculate the ratio of the M1/M2 phenotype of the macrophages in multiple myeloma tissues. Nuclei were labeled with 4',6-diamidino-2-phenylindole (DAPI). P65 antibody was used to label TAMs $\left(6 \times 10^{5}\right.$ cells/well $)$ as primary antibody, and FITC-conjugated goat anti-rabbit IgG (Beyotime, China) was incubated for secondary binding. DAPI was used to stain the nucleus. Fluorescence images were collected using an Eclipse 80i Microscope (Nikon, Japan).

\section{Cells and Maintenance}

We obtained H929 (human MM cells) from the Central Laboratory of the Affiliated Hospital of Nanjing University of traditional Chinese medicine. And the use of the cell line was approved by the Ethics Committee of the Affiliated Hospital of Nanjing University of Traditional Chinese Medicine. THP-1 (human monocytes) and SP2/0 (mouse MM cells) were purchased from iCell Bioscience (Shanghai, China). All cell lines were cultured in RPMI-1640 containing $10 \%$ FBS. The condition of humidifying incubator was $37{ }^{\circ} \mathrm{C}, 5 \% \mathrm{CO}_{2}$. In all experiments, cells were cultured to $80-$ $90 \%$ confluence and sub-cultured no more than 7 times.

\section{Indirect Co-Culture}

THP-1 monocytes were differentiated into macrophages by incubation with $50 \mathrm{ng} / \mathrm{mL}$ PMA for $24 \mathrm{~h}$ followed by incubation in RPMI 1640 medium for $24 \mathrm{~h}$. In the co-culture experiments, this differentiation occurred in six transwell inserts (membrane pore size of $0.4 \mu \mathrm{m}$ ). Transwell containing macrophages were co-cultured with H929 cells in a well plate for $16 \mathrm{~h}$ in $\mathrm{CO}_{2}$ independent medium supplemented with $3.75 \mathrm{~g} / \mathrm{L}$ of D-glucose and $0.5 \mathrm{mM}$ L-glutamine before being incubated with or without CRAE for $24 \mathrm{~h}$.

\section{Flow Cytometry}

Cell apoptosis was detected by Annexin V-FITC/propidium iodide (PI) double staining. The cells were inoculated in 6-well plates and treated with different doses of CRAE, or CRAE with TAK-242, and BAY 11-7085 when the density reached $6 \times 10^{5}$ cells/well. The cells were digested and collected, centrifuged at $1000 \mathrm{rpm}$ for $3 \mathrm{~min}$ to discard the supernatant. We washed the cells twice with $1 \times \mathrm{PBS}$ and suspended the cells again using $500 \mu \mathrm{L}$ of $1 \times$ binding buffer. Next, $10 \mu \mathrm{L}$ Annexin V-FITC and $10 \mu \mathrm{L}$ PI were added to cell suspension and incubated in darkness for 15 min. Finally, cells were detected by flow cytometry (BD, NJ, United States).

\section{Sorting of Macrophages Using Liberase-Based Perfusion Technique and MACS}

Macrophages of the mouse tumor tissue were sorted by magnetic cell sorting (MACS) in combination with the liberase-based perfusion technique and a low-speed centrifugation. We added Hank's balanced salt solution 5 times the volume of tumor tissue into $15-\mathrm{mL}$ centrifuge tube, then added liberase TM $0.3 \mathrm{mg} / \mathrm{mL}$ and DNAse $50 \mu \mathrm{g} / \mathrm{mL}$, mixed them well and incubated them in a shaker at $37^{\circ} \mathrm{C}$ for $1 \mathrm{~h} .5 \mathrm{~mL}$ of pre-cooled complete medium were added to neutralize the residual enzyme and the single-cell suspension were collected through 200 mesh steel into a new $15 \mathrm{~mL}$ centrifuge tube. The centrifuge was set at $4{ }^{\circ} \mathrm{C}$ and $300 \mathrm{~g}$ for $10 \mathrm{~min}$ and the supernatant was discarded to obtain tumor tissue single cells. Cells were resuspended in $450 \mu \mathrm{L}$ pre-cooled $1 \times \mathrm{PBS}$ and $50 \mu \mathrm{L}$ MASC F4/80+ microbeads were added and mixed. We placed it on a $4{ }^{\circ} \mathrm{C}$ freezer and incubated it for $15 \mathrm{~min}$. The LS column was placed on a magnetic stand, washed by $1 \mathrm{~mL}$ of running buffer and $500 \mu \mathrm{L}$ incubated single-cell suspension was added to the LS column, which was then washed by $2 \times 1 \mathrm{~mL}$ of running buffer. One milliliter of running buffer was added to the LS column and placed in a $15-\mathrm{mL}$ centrifuge tube. Sorted F4/80 + macrophages were quickly washed down with the back end of the syringe. The centrifuge temperature was set at $4{ }^{\circ} \mathrm{C}, 300 \mathrm{~g}$ for $5 \mathrm{~min}$. The supernatant was discarded to obtain $\mathrm{F} 4 / 80+$ macrophages for later experimental use.

\section{Western Blotting}

Proteins in the tumor tissues were extracted as follows: the tumor tissues of mice were chopped up and fully ground with RIPA lysis buffer containing protease inhibitors at low temperature. Then they were centrifuged at $15000 \mathrm{rpm}$ at 4 
${ }^{\circ} \mathrm{C}$ for $15 \mathrm{~min}$. We collected the supernatant and determined its concentration with BCA protein concentration determination kit. To obtain the cell proteins, after centrifugation, washing, RIPA lysis buffer was used to fully lysis the cell precipitate. The lysates were ultrasonicated and centrifuged and then we collected the supernatants for concentration determination. Protein lysates were denatured with sample loading buffer at $95^{\circ} \mathrm{C}$ and separated using sodium dodecyl sulfate polyacrylamide gel electrophoresis (SDS-PAGE). The proteins after separating were transferred to polyvinylidene difluoride (PVDF) membranes. The membrane was blocked with 5\% skimmed milk for 1 hour and then rinsed with TBST buffer for 3 times for 10 minutes each time. Membranes were incubated with primary antibodies overnight at $4{ }^{\circ} \mathrm{C}$. The secondary goat anti-rabbit horseradish peroxidase-conjugated antibody (cat\#ZB-2306) (ZSGBBIO, Beijing, China) was incubated at room temperature for $1 \mathrm{~h}$. Signals were quantitated using the Image Lab system, version 5.1.

\section{Molecular Docking}

The crystal structure of toll-like receptors 4 (TLR4) was derived from the Research Collaboratory for Structural Bioinformatics (RCSB) protein database (PDB ID: 2z63). Then, the protein structure was opened in Discovery Studio 2016 Client Software. The corresponding small molecular structure was downloaded from PubChem. Autodock Vina was used to dehydrate and hydrogenate small molecules and proteins. ${ }^{15}$

\section{Statistical Analysis}

SPSS 22.0 was used for statistical analyses. Measurement data were presented as mean and standard deviation. One-way or two-way analysis of variance was used to analyze the differences between groups. $\chi^{2}$-test was used for statistically analyzing numerical data. Pairwise comparison in multiple groups was conducted with Turkey method or Scheffe method. A p-value lower than $0.05(P<0.05)$ was considered as the differences have statistical significance.

\section{Results}

\section{CRAE Could Inhibit the Growth of Subcutaneous Tumors Xenotransplanted in Nude Mice}

In the traditional Chinese medicine treatment of multiple myeloma, the root of $C$. dahurica has been widely used. Our previous study found that Shengma Biejia decoction (SMBJD) which contains Cimicifugae rhizome, as a classic and traditional prescription of traditional Chinese medicine, could inhibit the growth of multiple myeloma and stimulate the recovery of hemogram. ${ }^{12}$ In order to clarify the effect of $C$. dahurica, the sovereign medicine in SMBJD on multiple myeloma, we carried out elaborated animal experiments (Figure 1). The results showed that after 14-day (Day 9-22) continuous administration, continuous 14 days of CRAE gavage and twice a week of bortezomib treatment, the mean tumor volume of CRAE group was significantly smaller than that of Vehicle group (Figure 1B and C). While the effect of bortezomib on inhibiting tumor growth was better than that of CRAE, the Combo group showed stronger effect in tumor inhibition. Besides, Kaplan Meier survival curve exhibited that CRAE could prolong the survival time of mice to a certain extent (Figure 1D).

To evaluate the safety of the CRAE, we concentrated on the weight and pathological variation of liver, spleen and kidney in mice. There were no significant changes in the body weight of mice for each group at the end of treatment $(\mathrm{n}=8)$. We stained the liver, spleen and kidney slices with hematoxylin and eosin staining. The spleen of the Vehicle group and each treatment groups showed a certain degree of white pulp injury and extramedullary hematopoiesis when compared with the blank group. There were mild glomerular mesangial matrix and cell proliferation in the kidney of the Vehicle group and the Combo group, and no obvious abnormalities were found in other groups. Compared with the blank group, there were slightly more inflammatory cells in hepatic sinuses in bortezomib treatment group and combined treatment group, however there was no obvious abnormality in CRAE group (Figure 1E). The above results suggested that CRAE was safe and effective and did not cause obvious liver, spleen, and kidney injury. Besides, the combined application of CRAE and bortezomib had a stronger inhibitory effect on tumor growth than bortezomib alone without serious organ injury. 
A

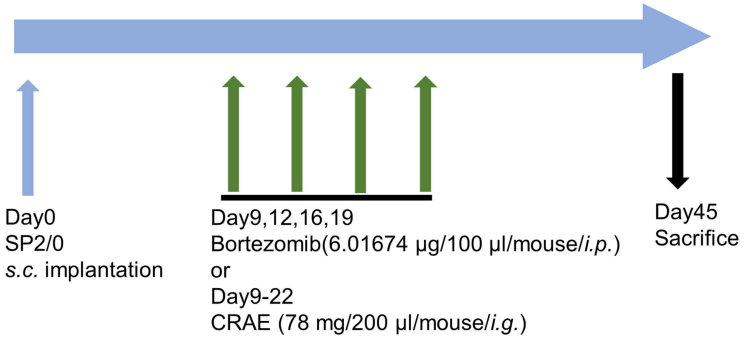

C

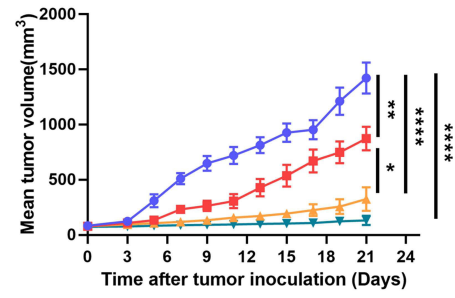

E
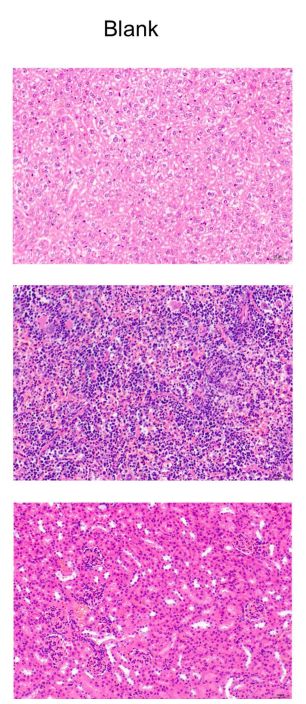

Vehicle
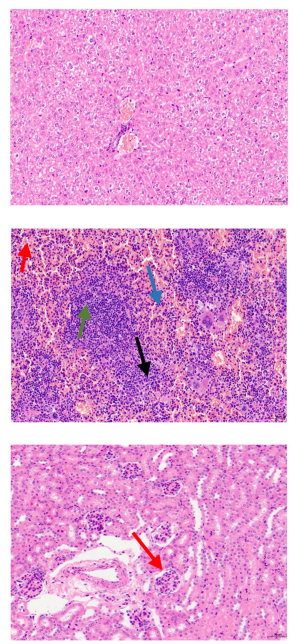
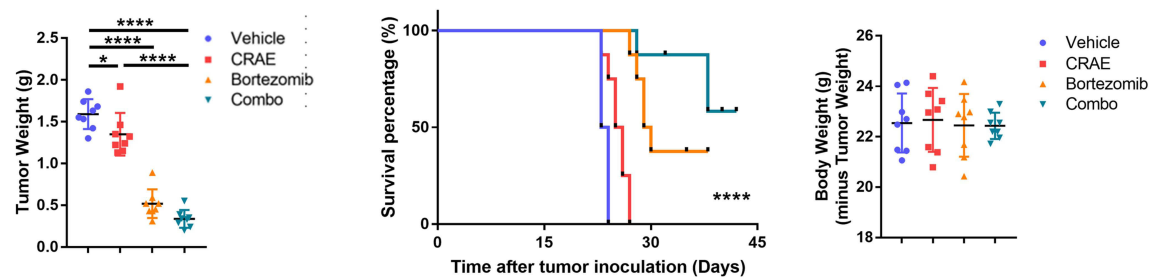

CRAE
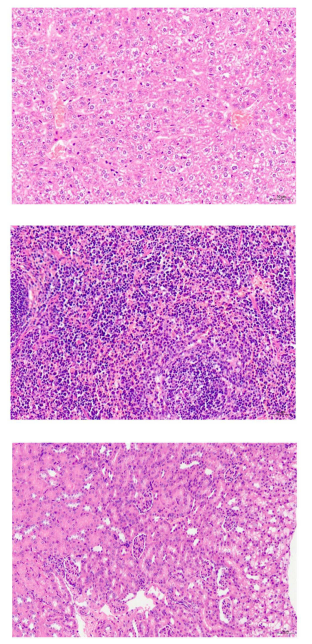

Bortezomib
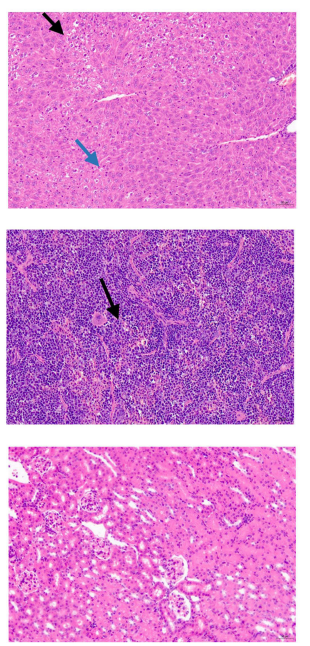

Combo
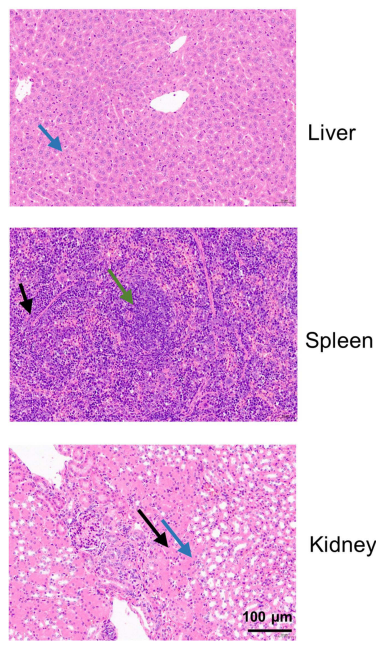

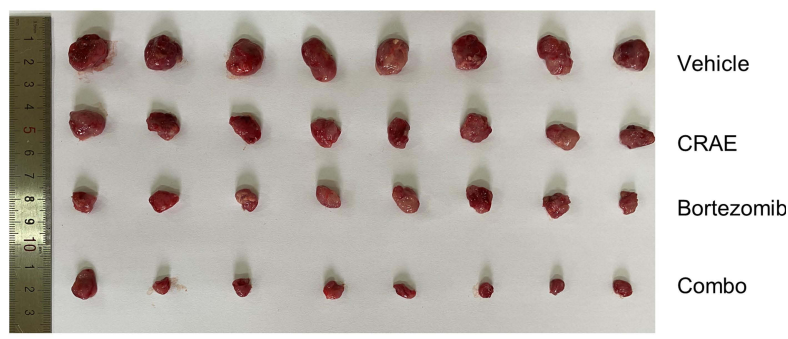

D

Figure I CRAE demonstrated its anti-tumor activity in the mouse xenograft model. (A) Time schedule of tumor implantation and drug therapy. (B) Measurement of tumor size. (C) Tumor volume and tumor weight. (D) Survival curves and body weight of different treatment types, such as Vehicle, CRAE, Bortezomib, or Combo treatment in SP2/0 xenograft mouse model ( $n=8$, one-way ANOVA or two-way ANOVA, $* P<0.05, * * P<0.01$, $* * * *<<0.000$ I). (E) Representative images of HE staining of same group mouse liver, spleen, and kidney. Magnification, $\times 200$. The black arrow in the pictures of liver refers to vacuolar degeneration and the blue arrow refers to inflammatory cell infiltration. The red arrow in the pictures of spleen refers to red pulp hemorrhage, the green arrow refers to white pulp, the blue arrow refers to mature neutrophils and the black arrow refers to extramedullary hematopoiesis. The red arrow in the pictures of kidney refers to mesangial cells proliferation, the black arrow refers to tubular atrophy and the blue arrow refers to inflammatory cells infiltration.

\section{CRAE Inhibited Myeloma Progression via TAM Remodeling in vivo and in vitro}

To explore the potential target of CRAE on tumor microenvironment, we carried out immunofluorescence. The results revealed that compared with the Vehicle group, the percentage of F4/80 + CD86 + cells was significantly risked in CRAE group and Combo group (Figure 2A). According to the immunohistochemical results, the expression levels of M2 macrophage markers (Arg1 and CD163) in CRAE group and Combo group decreased significantly, while the expression levels of M1 macrophage representative markers (iNOS and CD86) increased significantly (Figure 2B). These phenotypic changes were verified by WB. Macrophages of the mouse tumor tissue of four groups were isolated using MACS. Compared to the Vehicle group, each treatment group could increase the expression of CD86 and iNOS protein to a certain extent. Compared with Bortezomib group, the expression levels of CD86 and iNOS protein in CRAE group 
A
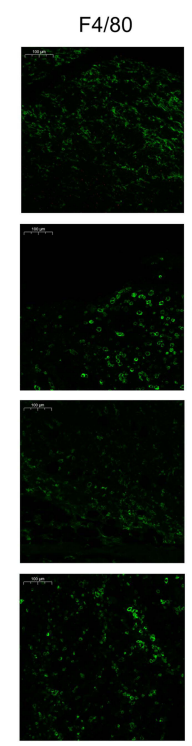
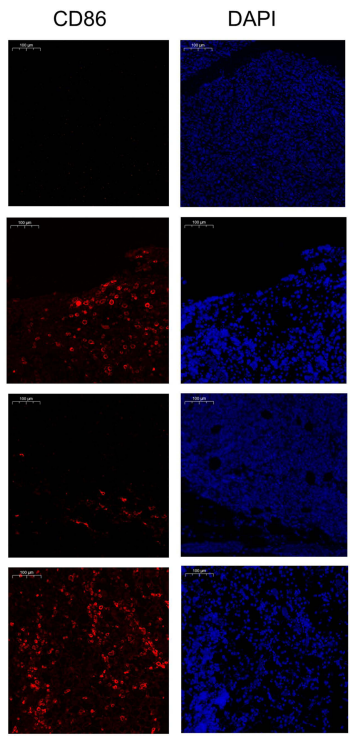
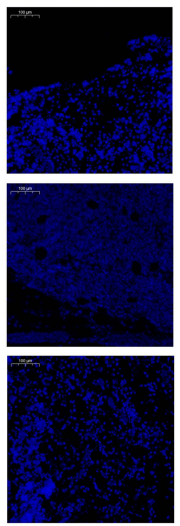

Merge
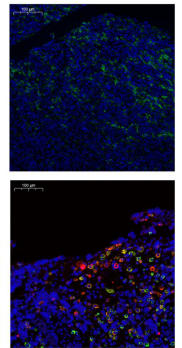

CRAE
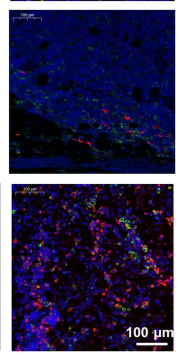

B

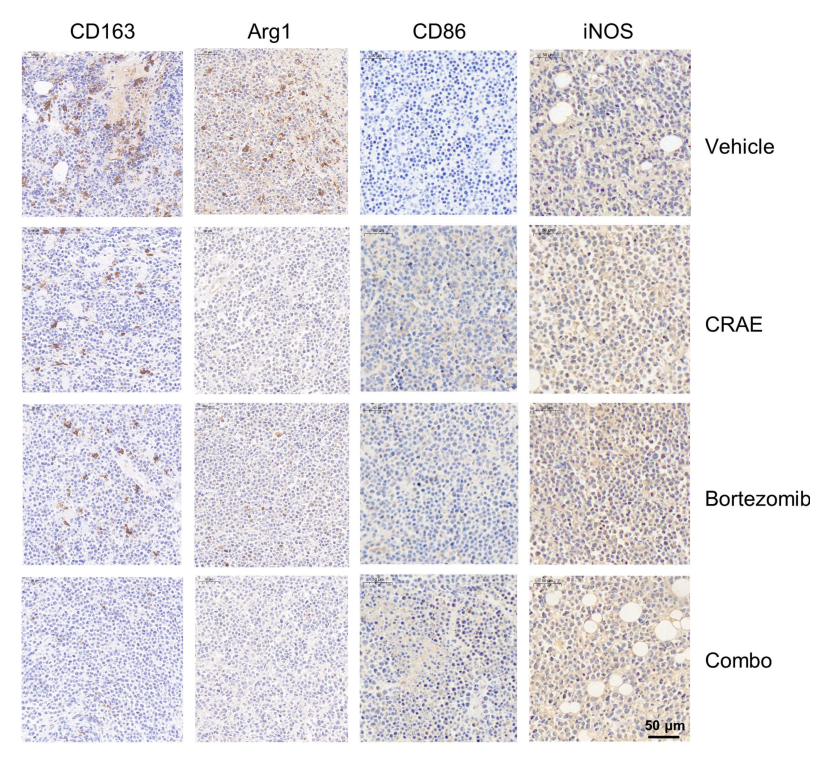

C

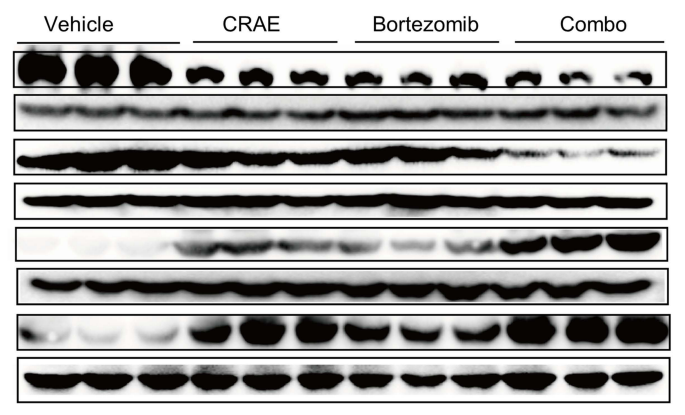

D
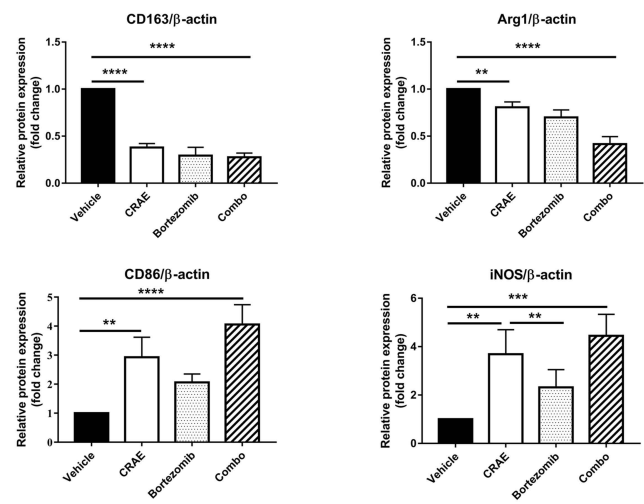

E
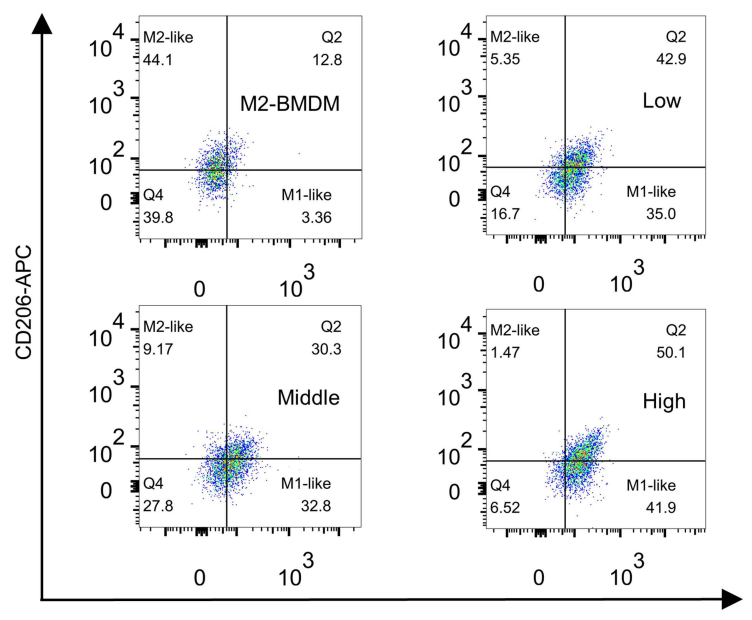

CD86-PE

$\mathbf{F}$
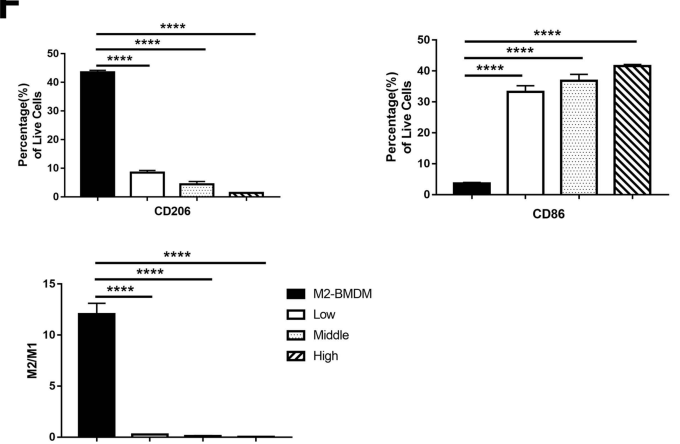

Figure 2 CRAE induced phenotypic switch of macrophages from M2 polarization to MI polarization in vivo and in vitro. (A) Detection of protein expression and localization of CD86 and F4/80 in tumor tissues were determined using an immunofluorescence assay. Magnification, $\times 100$. (B) Immunohistochemistry results showed that CRAE decreased the expression level of CDI63 and Argl, increased the expression level of CD86 and iNOS compared with the Vehicle group. Magnification, $\times 200$. (C) The expression levels of CD86 and iNOS (MI-like macrophage markers), and the expression levels of CDI63 and ArgI (M2-like macrophage markers) were detected by WB in vivo. (D) The expression of CDI63, ArgI, CD86 and iNOS levels of macrophages in tumor tissue are displayed as histograms (one-way ANOVA or two-way ANOVA, $* * P<0.01$, $* * * P<0.001$, $* * * * P<0.000 \mathrm{I}$ ). (E and F) Flow cytometry were used to detect the percentages of CD86+ cells or CDI63 cells of BMDM-M2 in vitro (****P< $0.0001)$. 
were more significant (Figure 2C and D). These data suggested that CRAE could inhibit the progression of myeloma by reducing M2-like macrophages and increasing M1-like macrophages in the tumor microenvironment.

For the purpose of exploring the regulatory effect of CRAE on macrophage polarization further, M2-like BMDMs were treated with or without CRAE. Flow cytometry displayed that CRAE treatment group could significantly increase the expression level of CD86 and reduce the expression level of CD206 in M2-like BMDMs (Figure 2E and F). ELISA analysis showed that compared with BMDM-M2 medium, the level of TNF- $\alpha$ and IL-12 in M2 macrophage medium

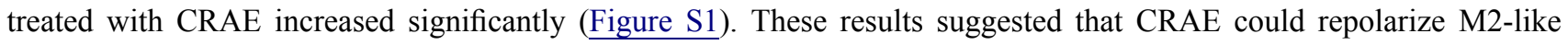
macrophages into M1 phenotype.

\section{Removal of Macrophages Attenuated the Antitumor Effect Mediated by CRAE}

As we found that CRAE had a regulatory efficacy on the characteristics of myeloma macrophages in animal experiments, macrophages were effectively cleared in mice by intraperitoneal injection of chlorophosphate (CL) to further observe the antitumor effect of CRAE. Meanwhile, mice injected with negative control PBS liposome (PL) were set as the control group. The mice were euthanized on the $23 \mathrm{rd}$ day, the tumor tissues were stripped and weighed (Figure 3A). The experimental results showed that the tumor weight and the tumor volume were significantly lower in both CRAE group and CRAE+PL group than that in the Vehicle group (Figure 3B-D). When macrophages were depleted, the effect of CRAE on inhibiting tumor growth was significantly weakened. Compared with the Vehicle group, the body weight of the CRAE group and CRAE+PL group was slightly higher $(P<0.05)$ (Figure $3 \mathrm{E})$.

\section{CRAE Alleviated the Unfavorable Differentiation of TAMs in Co-Culture System}

In order to explore whether CRAE could exert antitumor effect by affecting tumor stromal cells such as macrophages, we used transwell chamber to indirectly co-culture THP-1 induced by PMA with H929 and treated with or without CRAE (Figure 4A and B). Macrophages in each group were collected for follow-up analysis. The cell morphology showed that the normal THP-1 cells had full morphology and good refraction and were single-cell suspension growth. When THP-1 were stimulated by PMA phorbol ester for 24 hours, all the cells adhered to the wall in a round or spindle shape. After being co-cultured with myeloma cells, macrophages showed a tendency of aggregation and adherent growth and some of them extended pseudopodia, but there were relatively few long spindle cells. After CRAE treatment, the morphology of TAMs changed, pseudopodia extended and the cells were long spindle shaped (Figure 4C). Flow cytometry showed that the proportion of CD86-positive cells increased while the proportion of CD163-positive cells decreased in CRAE treatment group (Figure 5A). Western blotting demonstrated that compared with the co-culture control group, the levels of M1 characteristic proteins, iNOS and CD86 increased, while the levels of M2 characteristic proteins, CD163 and Arg1 decreased (Figure 5B and C). Immunofluorescence results also verified the changes of protein levels (Figure 5D).

\section{CRAE Regulated Macrophage Polarization by TLR4-NF-אB Pathway}

TLR4, p65, p-p65 and IL-1 $\beta$ proteins of macrophages isolated from tumor tissues were detected quantitatively. As shown in Figure S2, CRAE up regulated the expression of TLR4, p-p65 and IL-1 $\beta$. We examined the expression of key proteins of TLR4-NF- $\mathrm{BB}$ signaling pathway in groups of co-culture systems. The results showed that the levels of TLR4 protein and TLR4 junction protein-myeloid differentiation primary response 88 (MyD88) and TGF- $\beta$ activate kinase 1 (TAK1) phosphorylation increased in CRAE-treated group. We further analyzed the downstream phosphorylated protein, $p$-IKK $\beta$, $\mathrm{p}-\mathrm{I \kappa B}$, and p-p65. The results showed that the level of phosphorylated protein in CRAE treatment group was significantly higher than that in the control group (Figure 6A and B). p65 is the main effector protein of nuclear factor-kappa B (NF$\mathrm{Kb}$ ) signaling pathway, and the activation of NF- $\mathrm{KB}$ signaling pathway leads to NF- $\mathrm{KB}$ p65 translocation from cytoplasm to nucleus. We labeled p65 by immunofluorescence staining and detected the entry and exit of p65 of macrophage in the co-culture system by fluorescence microscope. We observed that in the co-culture control group, p65 was rarely expressed in the nucleus and had weak fluorescence expression in the cytoplasm. After CRAE intervention, the red fluorescence intensity of p65 increased and the expression in the nucleus increased significantly in a dose-dependent manner (Figure 6C). 
A

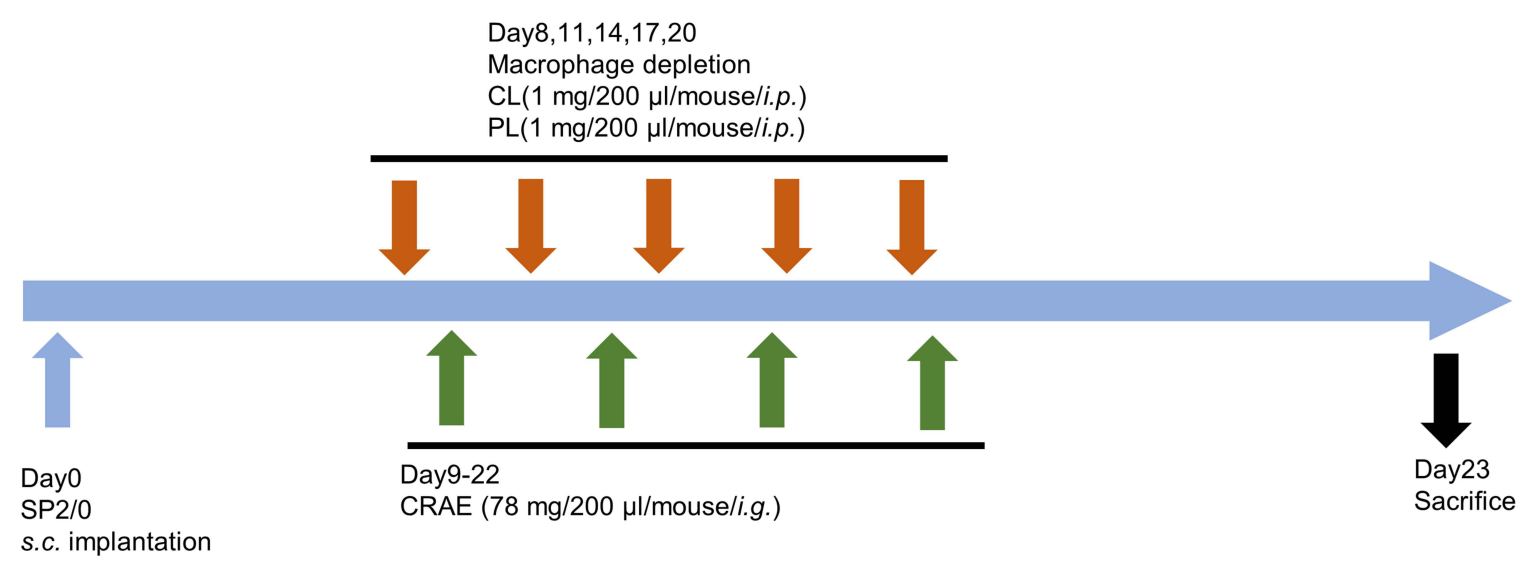

B

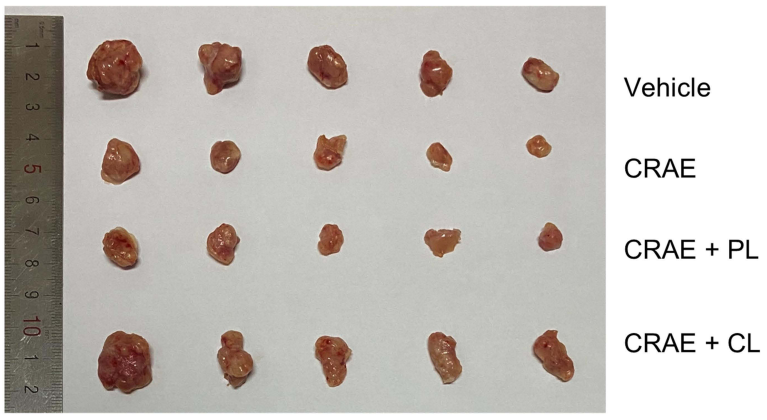

D

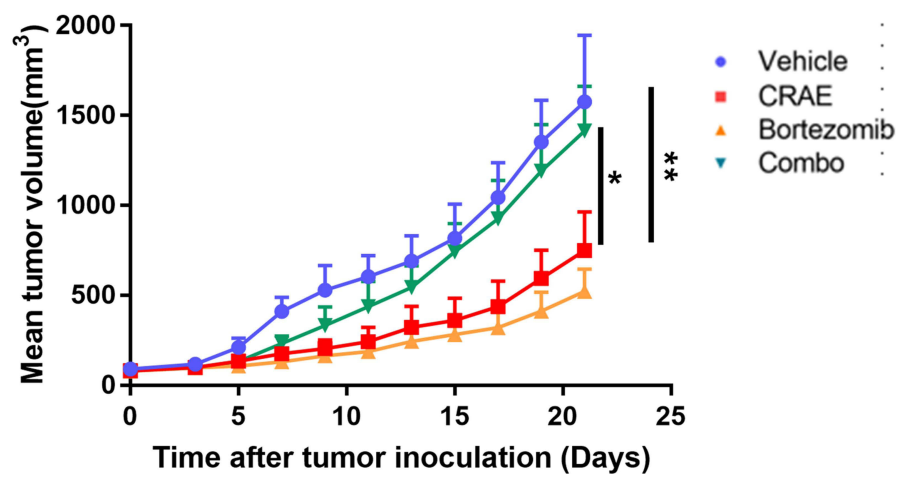

C

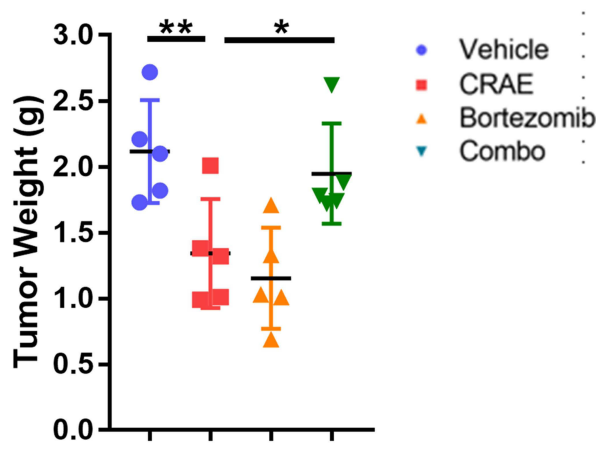

E

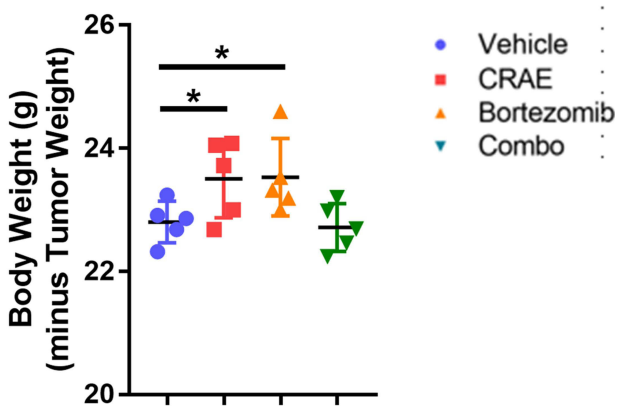

Figure 3 Macrophage depletion assay by clodronate liposome (CL) or negative control PBS liposome (PL). (A) Time schedule for tumor implantation and drug treatment. (B) Measurement of tumor size. (C) Tumor weight, (D) tumor volume, (E) body weight for different treatment types, Vehicle, CRAE, CRAE+PL, or CRAE+CL in SP2/0 xenograft mouse model $(n=5$, one-way ANOVA or two-way ANOVA, $* P<0.05, * * P<0.01$ ).

After performing TLR4 protein inhibitors TAK-242 and NF-KB inhibitor Bay 11-7085 (Bay), the decreasing effect of CRAE on CD163 and Arg1 protein was significantly reversed and the increasing effect of CRAE on CD86 and iNOS protein was also impaired (Figure 7A and B). Compared to the control group, the p65 red fluorescence of macrophages treated with TAK-242 and Bay was slightly enhanced, but its position was still mainly expressed in cytoplasm (Figure 7C). Overall, these results suggested that CRAE activated TLR4-NF- $\mathrm{KB}$ in macrophages in the co-culture system signaling pathway.

\section{CRAE-Treated Macrophages Induced Cancer Cell Apoptosis in Co-Culture System}

In order to determine whether macrophages treated with CRAE affected the activity of myeloma cells by inducing apoptosis, we treated the TAMs with or without CRAE separately, and re co-cultured them with H929 cells after fluid 


\section{A}

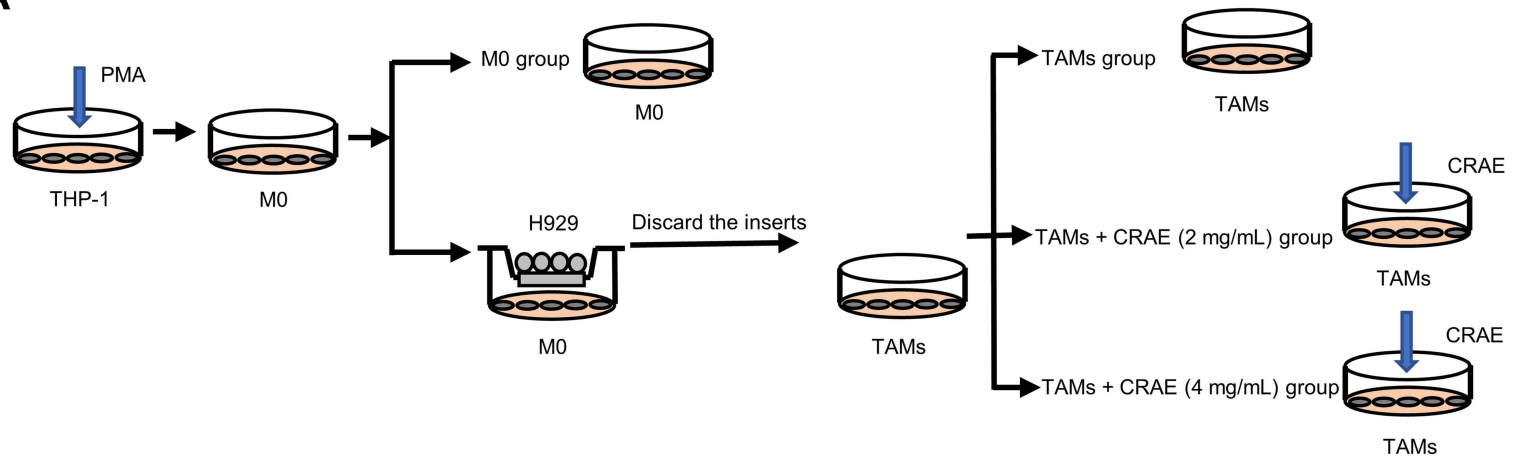

B

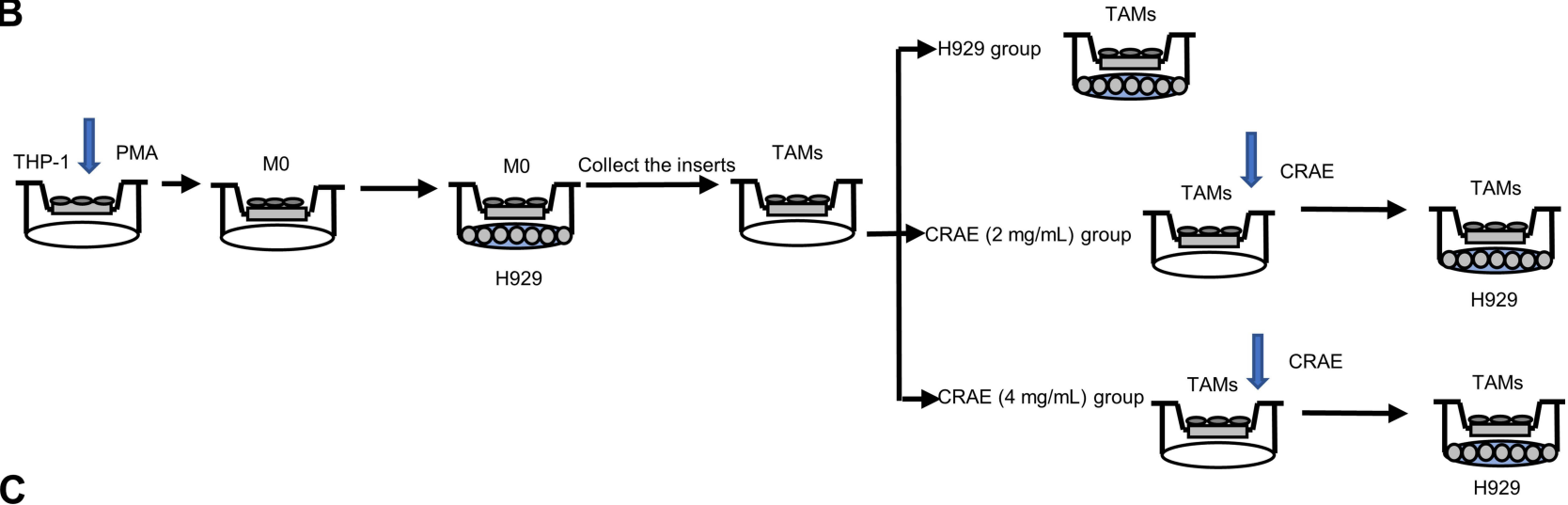

THP-1

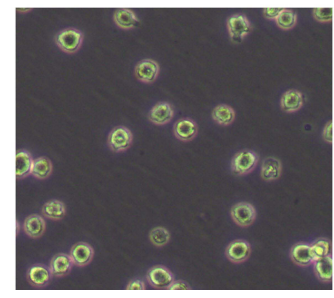

M0

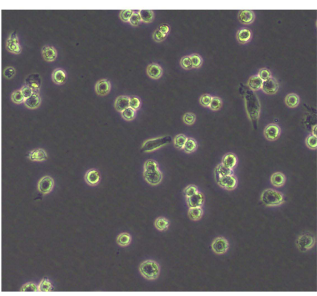

TAMs

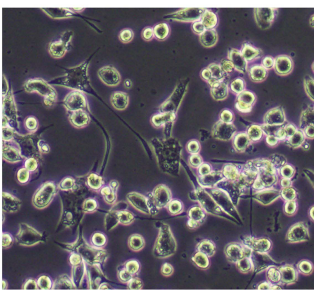

TAMs+ low dose

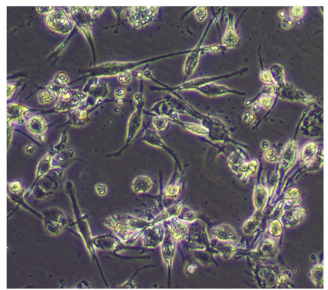

TAMs+ high dose

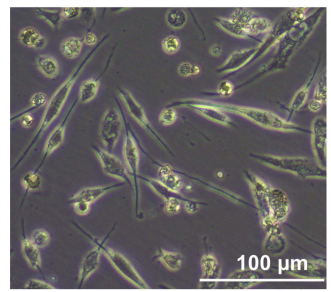

Figure 4 Grouping and treatment of cell models. (A) In vitro, PMA was co-culture with $\mathrm{H} 929$ cells to induce THP-I transformed into TAMs. Cells were grouped as follows: M0, TAMs, TAMs +CRAE $(2 \mathrm{mg} / \mathrm{mL})$, TAMs + CRAE $(4 \mathrm{mg} / \mathrm{mL})$. (B) To evaluate the anti-tumor effect of macrophages with or without CRAE, we first planted THP-I in the upper chamber of transwell chamber, co-cultured THP-I with $\mathrm{H} 929$ cells after the differentiation induced by PMA, then we took out the upper chamber separately, with or without CRAE, and re co-cultured them with $\mathrm{H} 929$ cells after fluid exchange. Cells were grouped as follows: H929, CRAE (2 mg/mL), CRAE (4 mg/mL). (C) Morphological changes of THP-I cells and macrophages induced by CRAE. Magnification, $\times 100$.

exchange (Figure 4B). We used Annexin-V/PI double staining to detect apoptosis. Flow cytometry manifested that the percentage of apoptosis of myeloma cells increased in a dose-dependent manner (Figure 8A). TUNEL staining analysis further detected the degree of DNA breakage caused by macrophages on apoptosis of myeloma cells. The results showed that TUNEL-positive cells increased significantly in CRAE treatment group (Figure 8C). The induction of apoptosis by macrophages could be significantly reversed by TAK-242 and BAY (Figure $8 \mathrm{~B}$ and D). These results suggested that the macrophages treated with CRAE could induce tumor cell apoptosis.

\section{Chemical Components in CRAE and Binding Site Analysis of the CRAE-TLR4}

High performance liquid chromatography was performed to study whether chemical components of CRAE were directly related to activation of TLR4. CRAE matched 813 chemical compositions in mzCloud and 173 of which had an optimal matching score greater than 70 points (Figure 9A). In order to further determine which chemical component may interact with TLR4, we first found all possible targets in 173 chemical compositions based on Swiss Target Prediction database. And we supplemented the possible components in combination with Traditional Chinese Medicine Systems 
A

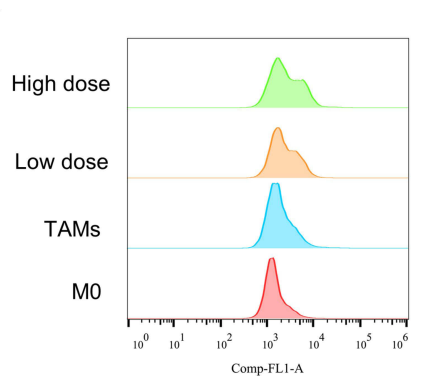

CD86 Alexa Fluor 488

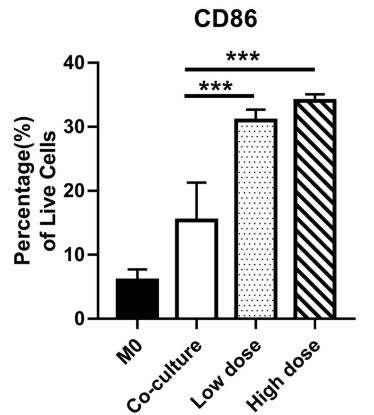

B

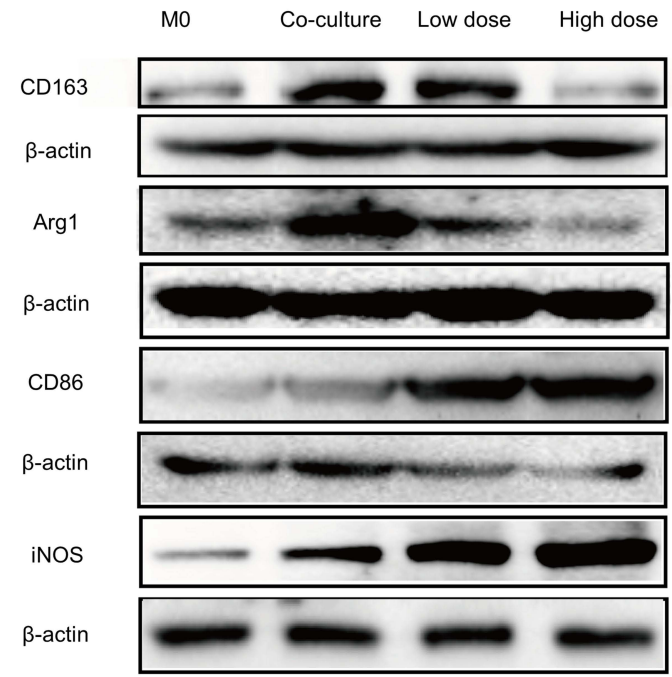

D

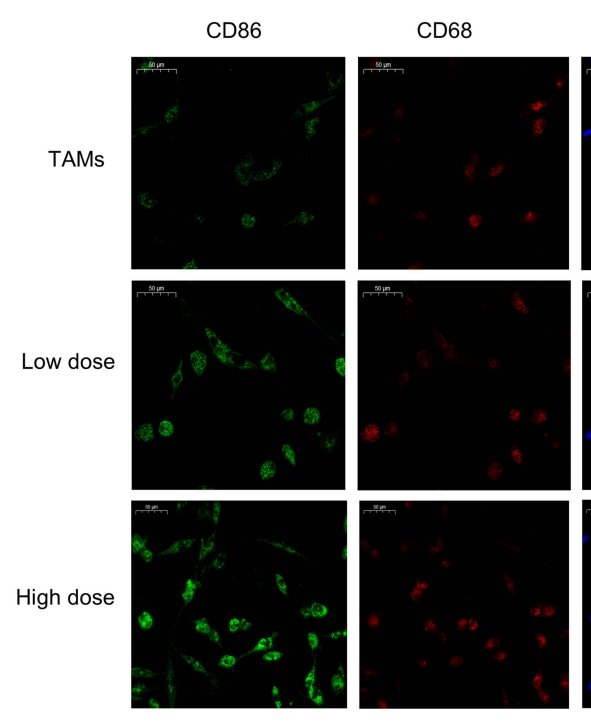

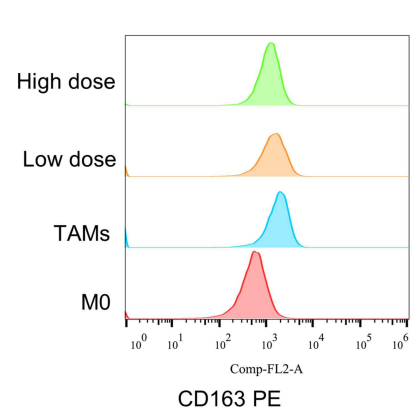

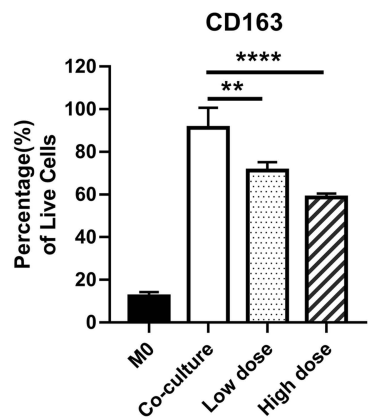

C
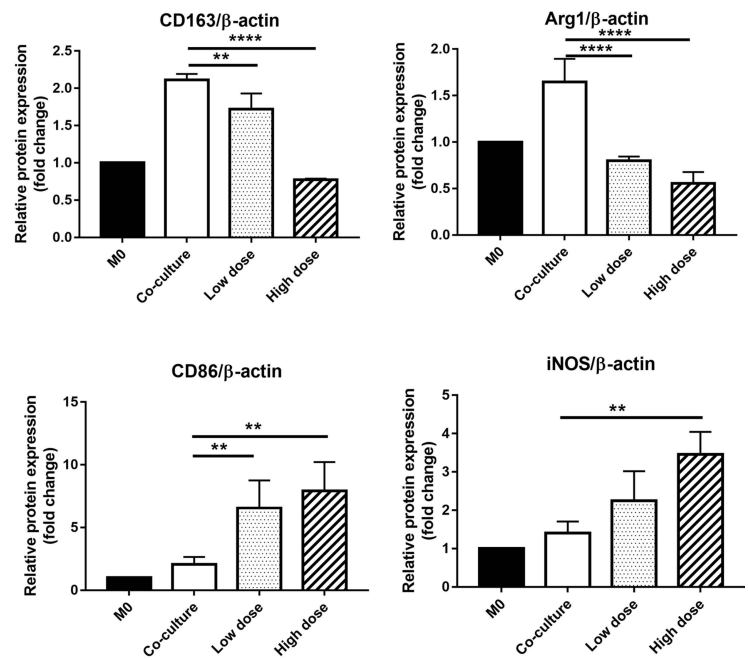
A

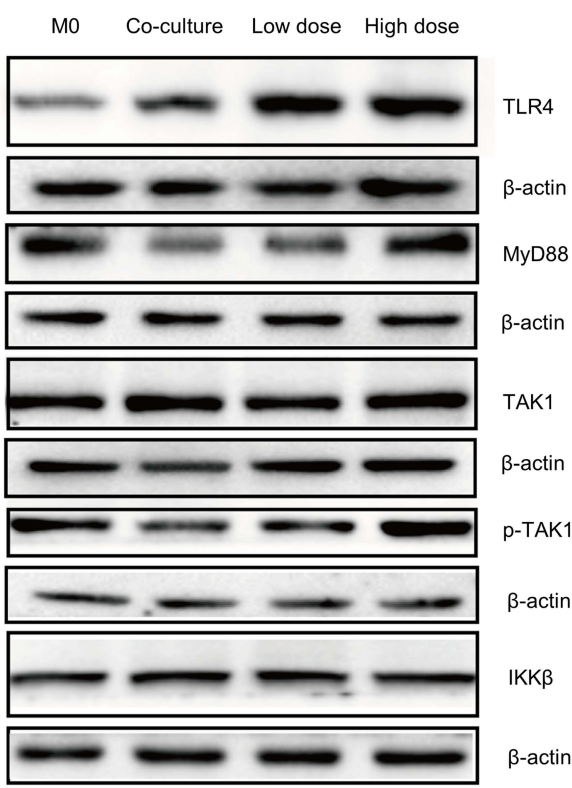

B

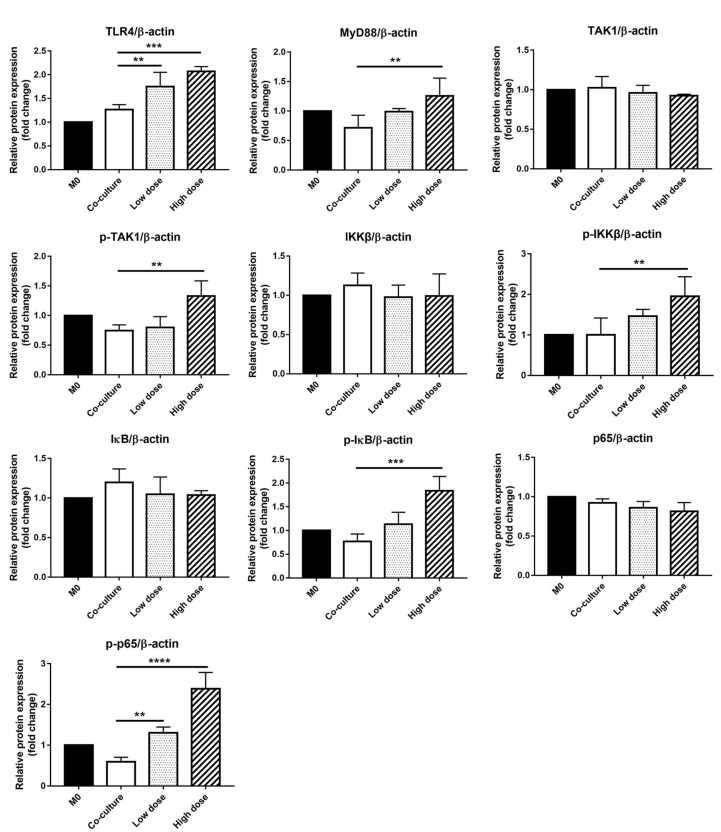

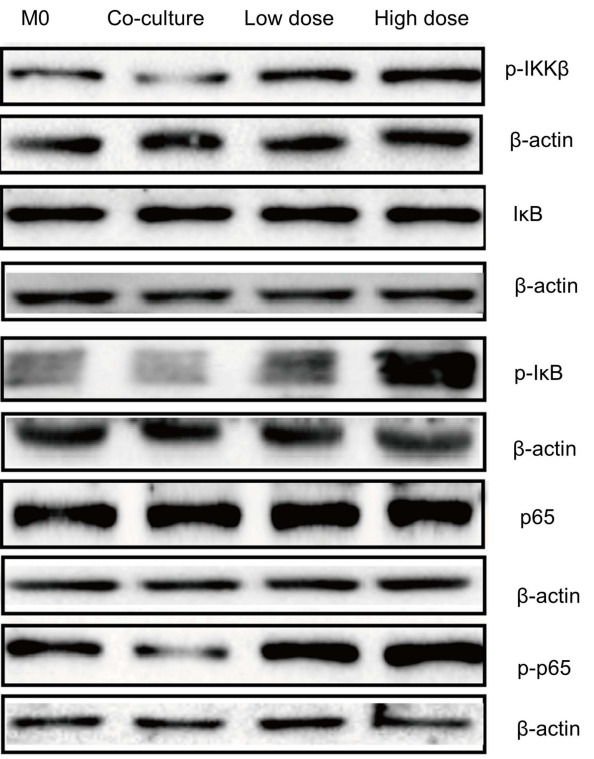

C

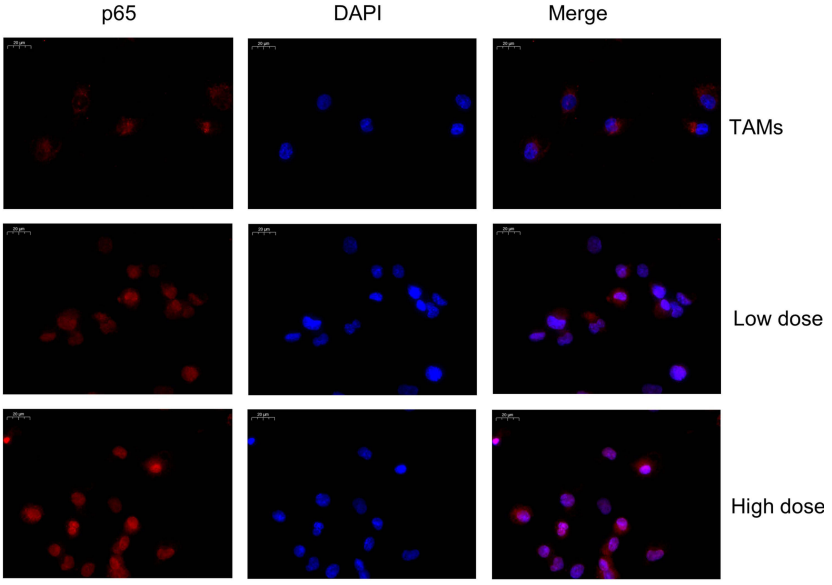

Figure 6 CRAE mitigated the unfavorable differentiation of TAMs via TLR4-NF- $K B$ signaling cascades. (A) Protein expressions of TLR4, MyD88, TAK-I, P-TAK-I, IKK $\beta$, $\mathrm{p}-\mathrm{IKK} \beta$, IкB, $\mathrm{p}-\mathrm{I} \mathrm{K}$, NF- $\kappa \mathrm{B}$, and $\mathrm{p}-\mathrm{NF}-\kappa \mathrm{B}$ in four groups were detected by WB. (one-way ANOVA or two-way ANOVA). (B) The corresponding expression of protein levels

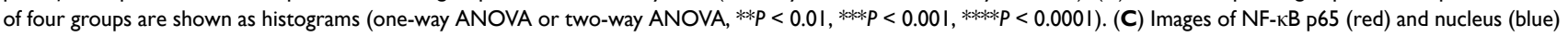
were obtained using a fluorescence microscope. Magnification, $\times 400$.

Pharmacology Database and Analysis Platform (TCMSP) database. It was gratifying that we found that 10 compounds ferulic acid (PubChem CID: 445858), 3,4-dimethoxycinnamic acid (PubChem CID: 717531), 3-methoxycinnamic acid (PubChem CID:637668), khellin (PubChem CID: 3828), sinapinic acid (PubChem CID: 637775), isoferulic acid (Pub Chem CID: 736186), caffeic acid (PubChem CID: 689043), 3-coumaric acid (PubChem CID: 637541), 5-(2-nitroprop1-enyl)-1,3-benzodioxole (PubChem CID: 5375839) and cimicifugic acid B (PubChem CID: 6449880) - could be bind to TLR4 (Table S1). Subsequently, we stimulated the binding patterns of TLR4 with these 10 compounds. As shown in 
A

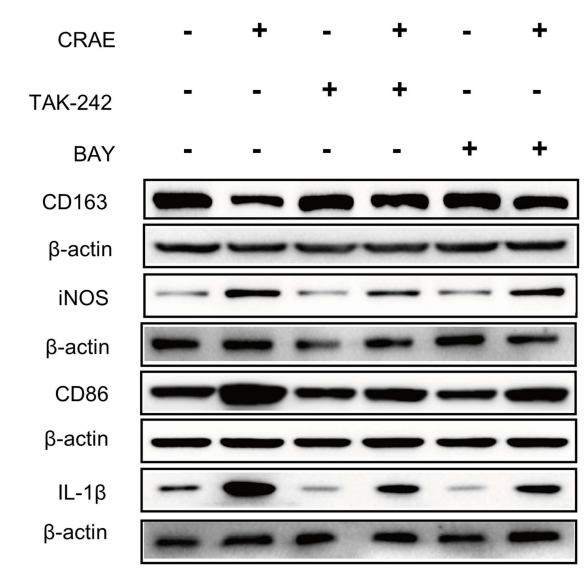

B
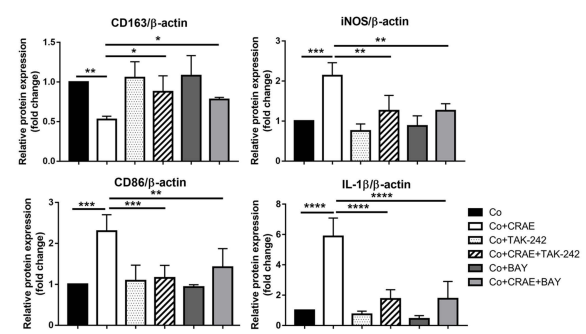

C

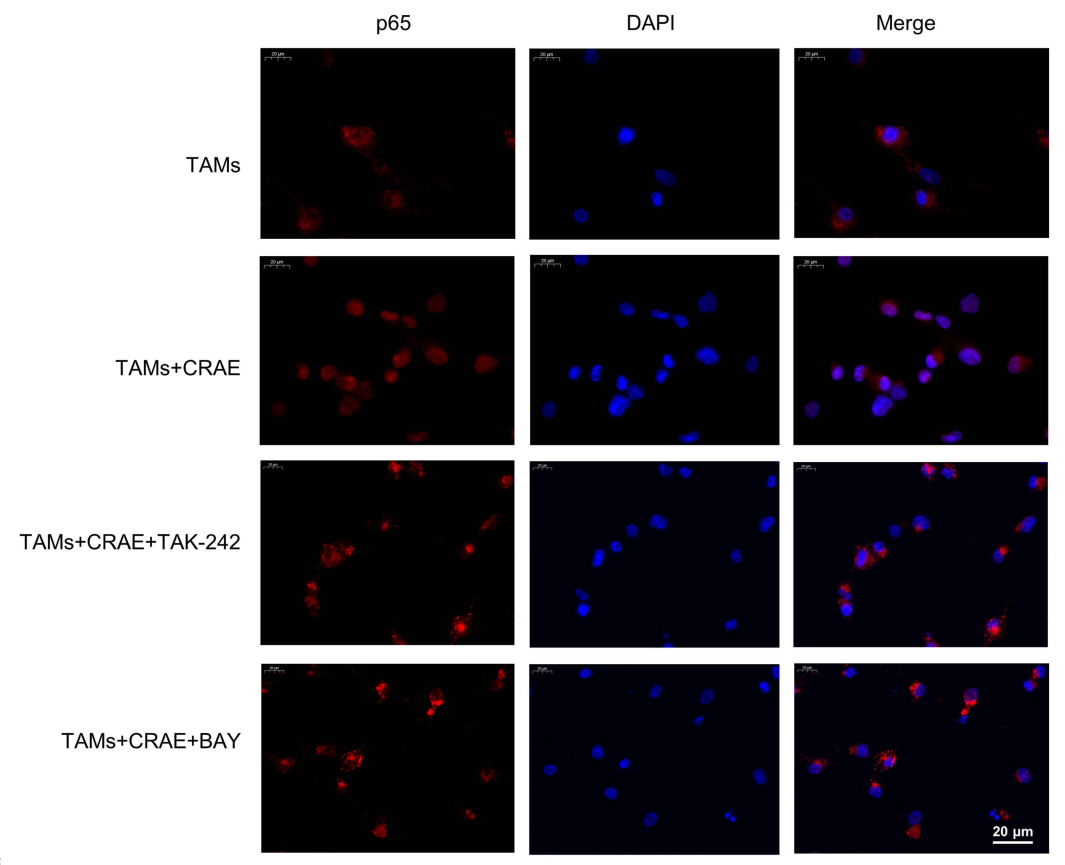

Figure 7 CRAE induced to the MI-type macrophage polarization and the MI-type polarization markers were inhibited by the TLR4 protein inhibitors TAK-242 and NF- $\mathrm{B}$ inhibitor Bay II-7085 (Bay). (A) Protein expression levels of MI macrophages markers and M2 macrophages markers were analyzed and semi-quantified through WB. (B) The corresponding expression of CDI63, iNOS, CD86 and IL-I $\beta$ levels are shown as histograms (one-way ANOVA or two-way ANOVA, $* P<0.05$, $* * P<0.0 \mathrm{I}$, $* * * P<0.00 \mathrm{I}$, $* * * * P<0.0001)$. (C) The expression level and localization of $\mathrm{p} 65$ protein in macrophages were detected by immunofluorescence assay. Magnification, $\times 400$.

Figure 9B-D, the structures, chromatogram and MS/MS diagrams of the three compounds were given: the highest binding free energy to TLR4 were cimicifugic acid B $(-6.9 \mathrm{kcal} / \mathrm{mol})$, caffeic acid $(-5.9 \mathrm{kcal} / \mathrm{mol})$ and isoferulic acid $(-5.7 \mathrm{kcal} / \mathrm{mol})$. The binding patterns of cimicifugic acid $\mathrm{B}$, caffeic acid and isoferulic acid with TLR4 are displayed in Figure 9E-G and the binding pockets of these on TLR4 were shown in Figure S4. Three hydrogen bonds were provided by the GLN 333, SER 352 and LEU283 residues in the interaction with cimicifugic acid B, whereas caffeic acid formed hydrogen bonds with SER 580, LYS 582, THR 553, GLY 585, ASN 554 of TLR4 and isoferulic acid interacted with TLR4 by forming hydrogen bonds with GLY 585, LYS 582, ASN 554, THR 553. In addition, the structures and binding patterns of the left seven are shown in Figure S3A-J. The results illustrated that many compounds in CRAE had binding sites to TLR4, which may affect the stability or activity of TLR4 (Figure 10).

\section{Discussion}

It has been confirmed that the survival and proliferation of malignant plasma cells in the bone marrow microenvironment depend on nonmalignant stromal cells. The interaction plays an important role in mediating drug resistance and maintaining minimal residual lesions, and ultimately affects the clinical behavior and prognosis. ${ }^{5,16}$ TAMs have a phenotype similar to M2-like macrophages and play a role in inhibiting adaptive immunity, promoting tumorigenesis, and angiogenesis. Infiltrating macrophages usually change the phenotypic characteristics when adapting to a series of activated states. ${ }^{17,18} \mathrm{CD} 68$ is a universal marker of macrophages. M2-polarized macrophages highly express arginase-1 (Arg-1), resistin-like molecules $\alpha$ (RELM $\alpha$ ), scavenger receptor (CD163) and mannose receptor (CD206). Unpolarized macrophages could be activated by Th1 cytokines, such as bacterial components (such as lipopolysaccharide), tumor necrosis factor (TNF- $\alpha$ ), and interferon (IFN- $\gamma$ ), and then alter its polarization state to M1 subtype which highly express TNF- $\alpha$, inducible nitric oxide synthase (iNOS), IL-6, IL-12, IL-23 and CXCL11 and exhibit anti-tumor activity. ${ }^{19,20}$ By 


\section{A}
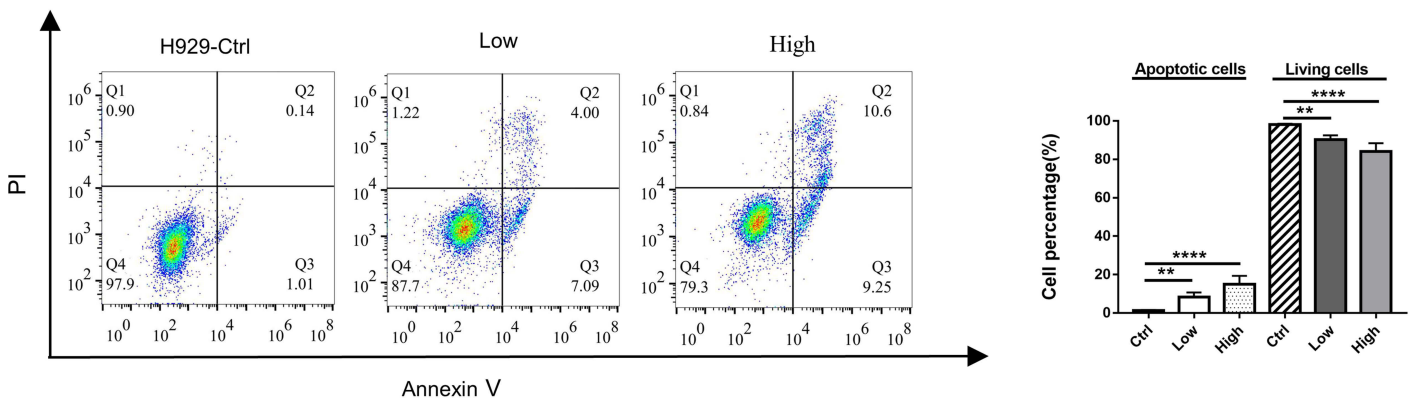

B
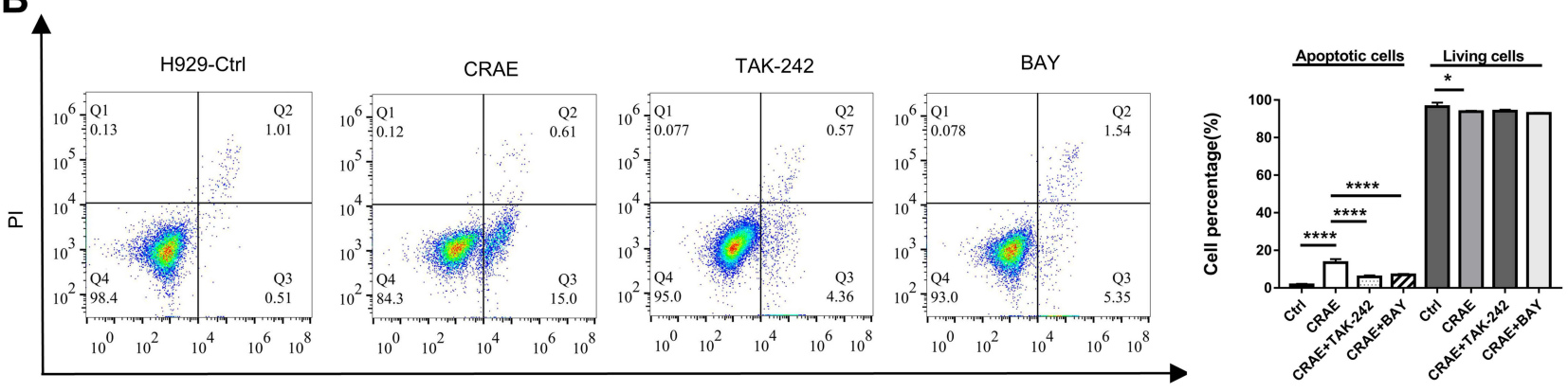

Annexin V

C

TUNEL
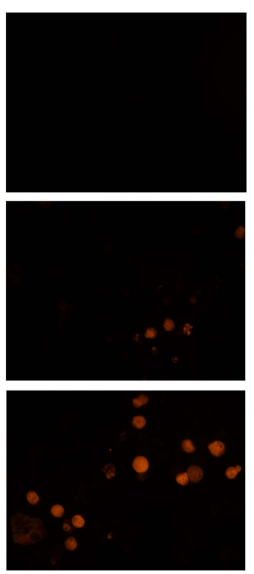

DAPI
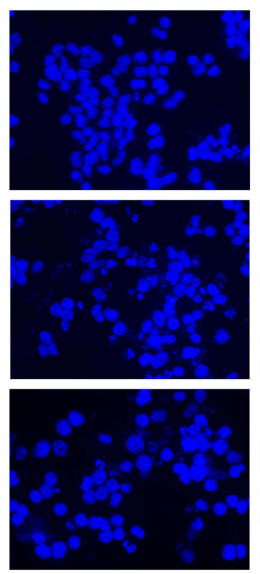

Merge
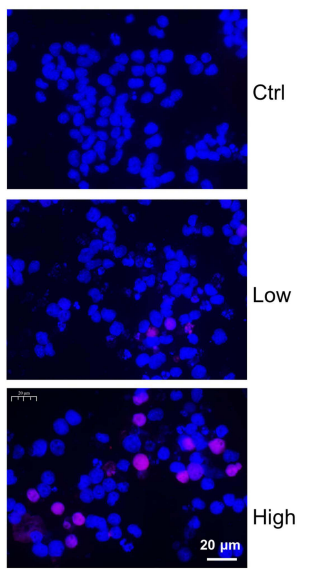

D

TUNEL
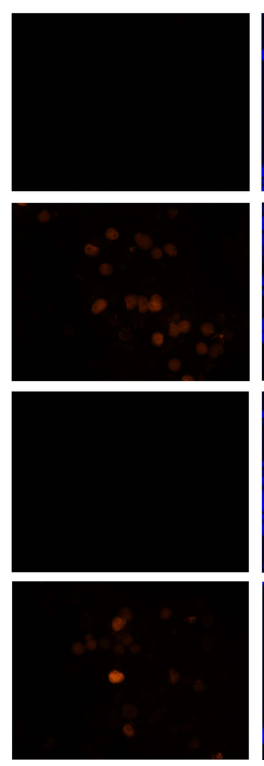

DAPI
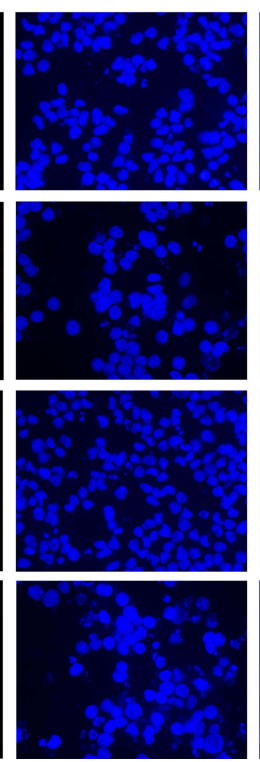

Merge
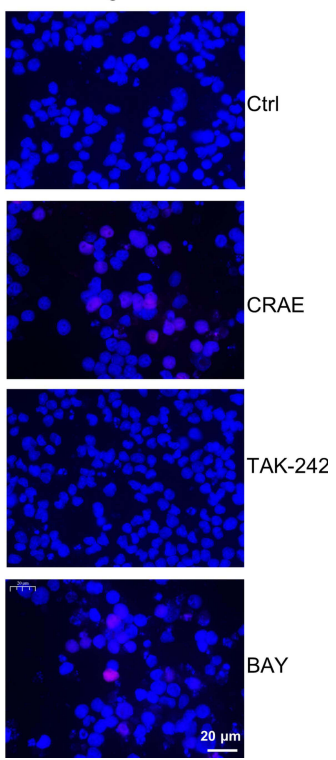

Figure 8 Modulation of $\mathrm{H} 929$ cell apoptosis induced by CRAE by co-cultured with TAM macrophages. (A) After incubating with macrophages, apoptosis of H929 cells was detected by flow cytometry (Annexin V -FITC and PI double staining) $(* * P<0.0$ I, $* * * * P<0.000 \mathrm{I})$. (B) The apoptosis of H929 cells with the treatment of CRAE, or CRAE + TAK-242, or CRAE+BAY was detected. The percentage of cells in each quadrant was calculated $(* P<0.05$, ****P $<0.000 \mathrm{I})$. (C) TUNEL assay detected apoptosis of DNA degradation in CRAE treatment. (D) The apoptosis of DNA degradation in CRAE treatment, or CRAE+TAK-242, or CRAE+BAY was detected. Magnification, $\times 400$.

evaluating the content of CD163+ TAMs in 240 patients with MM through IHC, Chen et al demonstrated that the M1/M2 ratio of TAMs was a new independent adverse prognostic factor for $\mathrm{MM}^{21}$ Another study based on myeloma patients treated with bortezomib attained the same results. It also suggested that TAM might be related to the development of MM resistance to bortezomib-based chemotherapy. ${ }^{22}$ In addition, our previous meta-analysis confirmed that macrophage derived soluble CD163 levels in a variety of cancers, including MM, predicted poor overall survival (OS) and 
A

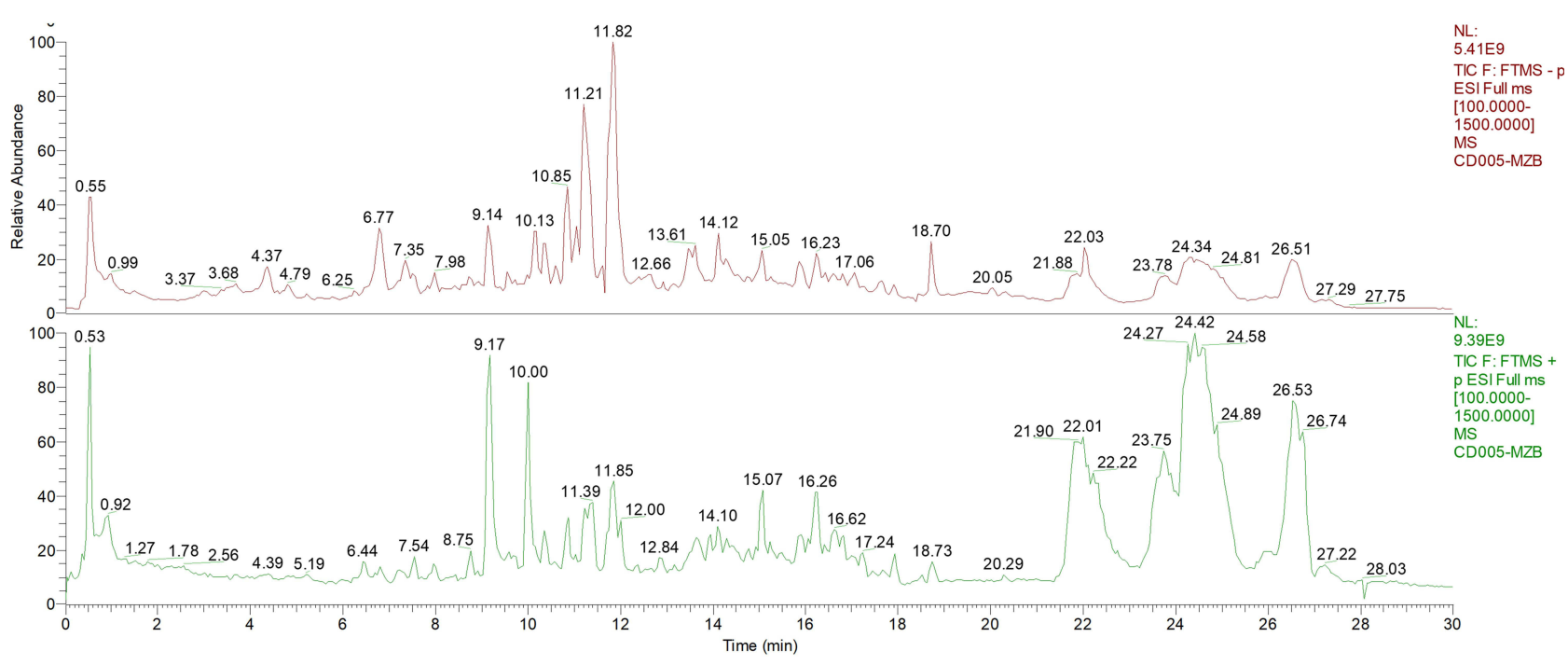

B<smiles></smiles>
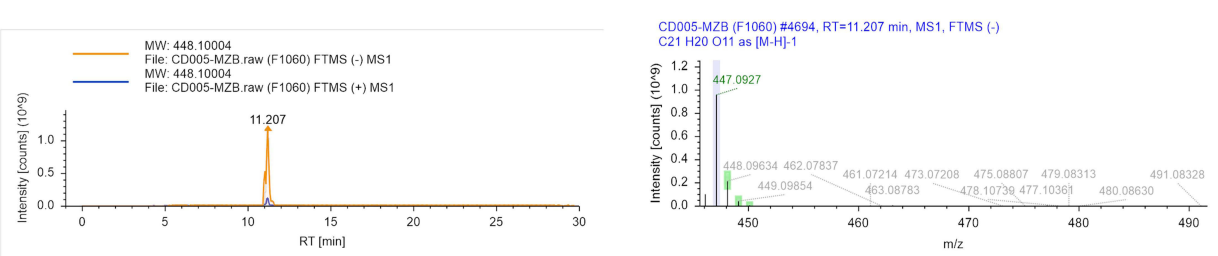

C<smiles>O=C(O)/C=C/c1ccc(O)c(O)c1</smiles>
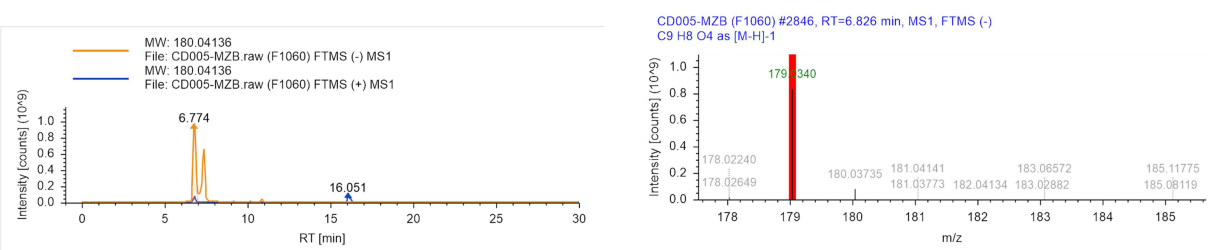

\section{D}<smiles>COc1ccc(/C=C/C(=O)O)cc1O</smiles>
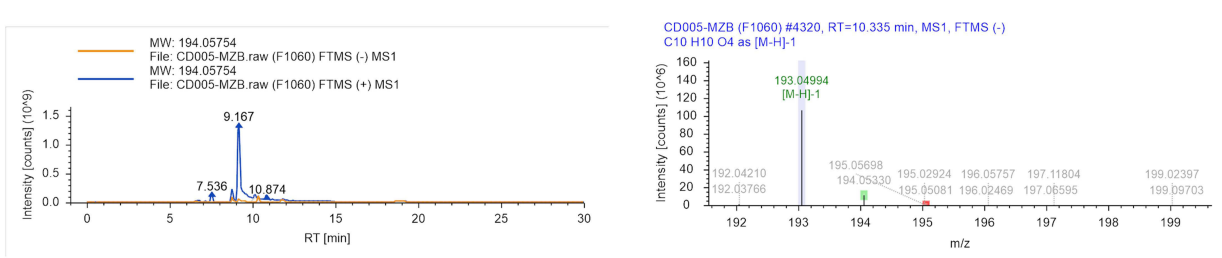

E

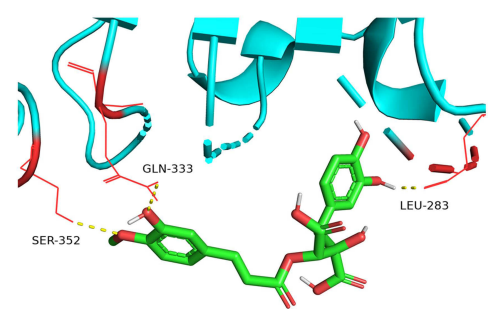

$\mathbf{F}$

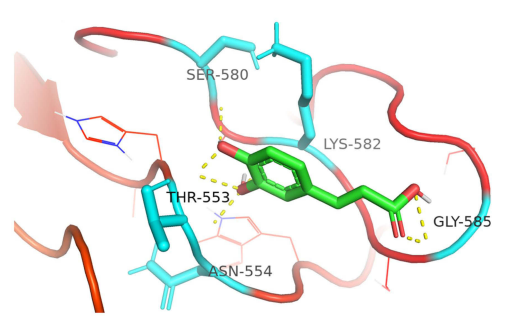

G

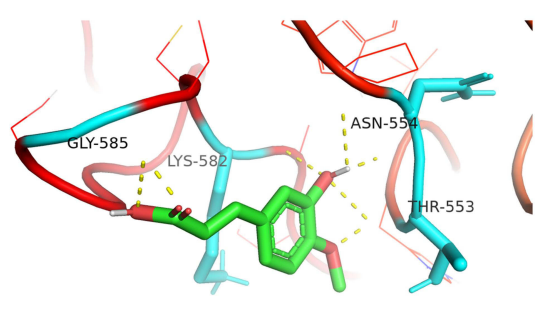

Figure 9 Chemical components of CRAE and binding site analysis of the CRAE-TLR4. (A) Total ion chromatograms of CRAE (green-negative mode, red-positive mode). (B-D) Chemical structures, chromatograms and MS/MS diagrams of cimicifugic acid B (B), caffeic acid (C), and isoferulic acid (D). (E-G) The potential binding sites of TLR4 and cimicifugic acid $B(\mathbf{E})$, caffeic acid $(\mathbf{F})$, and isoferulic acid $(\mathbf{G})$ were calculated by computer. 


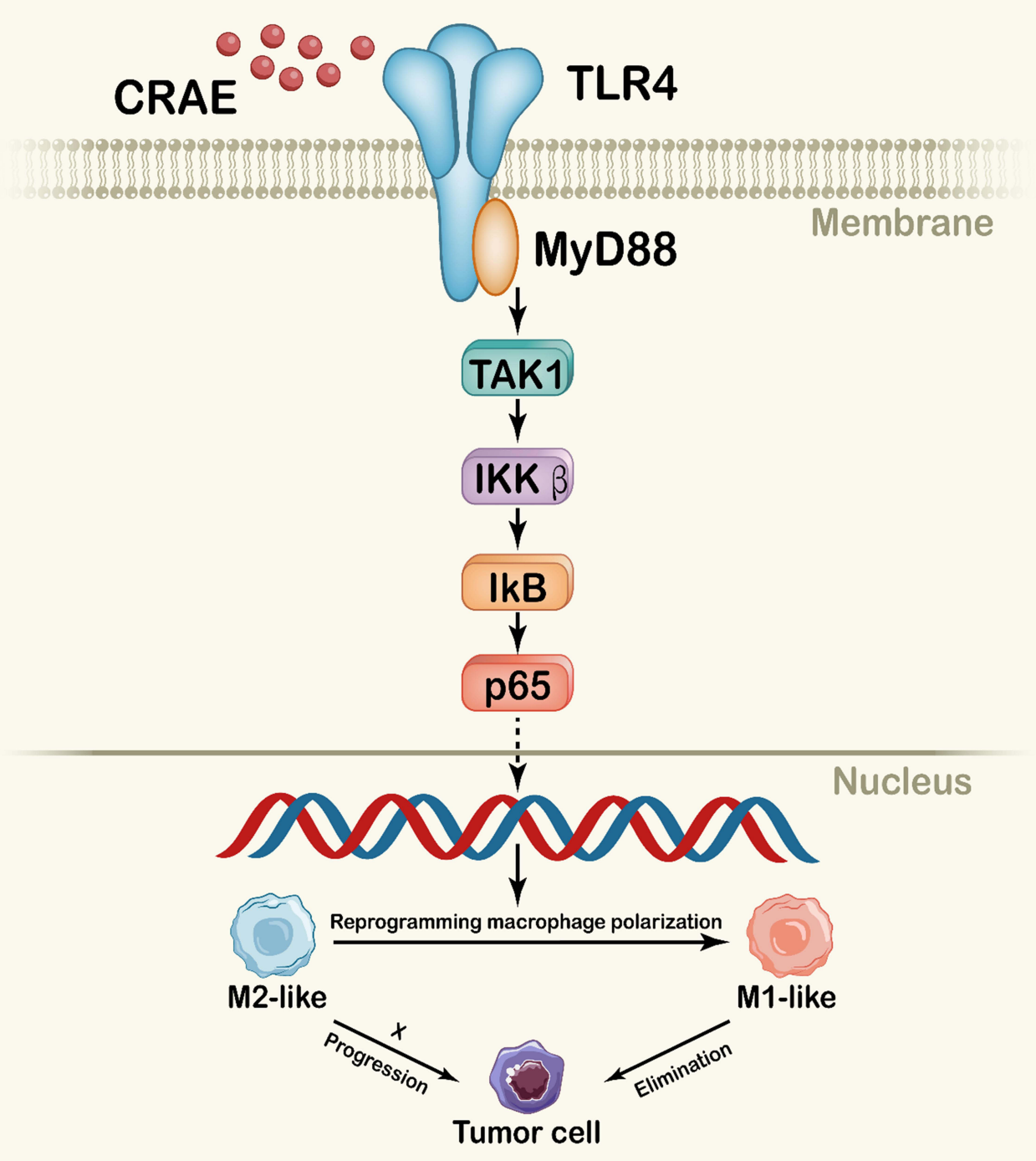

Figure 10 Schematic diagram of CRAE reprogramming macrophage polarization through TLR4-MyD88-TAKI-NF-KB axis. The active ingredient of CRAE caused the activation of TLR4, leading to NF-KB activation, promoted the reprogramming of macrophages, and then induced the apoptosis of myeloma tumor cells.

progression-free survival (PFS). ${ }^{23}$ In consideration of the vital role of macrophages in the occurrence and development of myeloma, the development of drugs targeting tumor microenvironment, especially macrophages, is worthy of research and expectation. Existing clinical studies have reported the regulatory effect of herbal medicines on the immune system of tumor patients. Studies have shown that medicinal herbs and their derivatives produce anti-cancer effects by regulating the polarization of macrophages in the tumor microenvironment. ${ }^{24,25}$ Our data showed that CRAE inhibited tumor growth in myeloma xenograft model and had a good combined therapeutic effect with bortezomib. Through a series of studies, we proved that CRAE could increase the expression of M1-specific indicators in tumor microenvironment and confirmed the activation of TLR4-NF- $\mathrm{KB}$ pathway was the main internal mechanism of CRAE mediated macrophage remodeling to inhibit the growth of myeloma.

Proteasome inhibitor bortezomib is one of the most effective treatments for MM. No studies have shown that proteasome inhibitors can regulate the polarization of myeloma-associated macrophages. It is generally believed that 
bortezomib can inhibit the activation of NF-kB signaling pathway and it has been used to treat inflammation, ${ }^{26-29}$ but few literatures reported the effect of bortezomib on tumor-associated macrophages. According to the existing literature, the interaction between bortezomib drug response and macrophages may be coexisted. It has been reported that myelomaassociated macrophages mediate the bortezomib-resistance of MM cells. ${ }^{8}$ And some studies showed that macrophages may also be the target of the synergistic effect of bortezomib and zoledronic. They synergistically affected the proliferation, adhesion and migration of MM macrophages, as well as their angiogenesis characteristics. ${ }^{30} \mathrm{Kim}$ et al demonstrated that the decreased proliferation index of the tumor cells under co-cultured with bortezomib pretreated macrophages. ${ }^{31}$ Current tumor treatment strategies targeting macrophages can be divided into four main groups: 1) inhibition of macrophages recruitment to the tumor, 2) direct restriction on TAMs, 3) transformation of TAMs from M2like pro-tumoral phenotype to M1-like anti-tumor phenotype, and 4) TAMs-mediated therapeutic substance delivery. ${ }^{16}$ In our study, bortezomib and CRAE may have different therapeutic function on macrophages in myeloma tumor environment. In vitro assay indicated that bortezomib did not show the effect of polarizing macrophages, although to some extent, bortezomib could reduce the infiltration of macrophages in vivo to reduce the effect of TAMs on MM. Our results showed that CRAE had an obvious polarization effect on myeloma-derived macrophages, which may become the possible reason for the better effect of bortezomib when combined with CRAE.

Studies have shown that macrophages can prevent melphalan-induced apoptosis of myeloma cells by inhibiting the activation of poly(ADP ribose) polymerization (PARP) and caspase-3 and maintaining the expression levels of Bcl-XL. ${ }^{32}$ Previous studies verified that M1-like macrophages can increase the apoptosis of tumor cells, while the proportion of apoptotic tumor cells co-cultured with M2 macrophages is lower than that in control cells, and the levels of cleaved PARP-1 and caspase-3 proteins are lower. ${ }^{33}$ In this study, in order to investigate the effect of CRAE-treated macrophages on the survival of myeloma cells, macrophages were treated with CRAE alone. The culture medium was refreshed to avoid the direct effect of CRAE on myeloma cells to affect the accuracy of the results. The results showed that in the indirect co-culture system with myeloma cells, CRAE-treated macrophages could increase the apoptosis of myeloma cells, and the apoptosis rate could be reversed by TAK-242 and BAY. To some extent, the apoptosis was due to the macrophage repolarization to M1-like subtype by TLR4-NF- $\mathrm{kB}$ pathway.

Toll-like receptors (TLR) exist in various cells and tissues. Many drugs activate antigen-presenting cells including macrophages through TLR. Some TLR-associated agonists have potential anti-tumor function by activating innate antitumor immune response. TLR4 signals can be transmitted through MyD88 and TRIF pathways. MyD88 pathway induces the early activation of NF- $\mathrm{KB}$ by recruiting IL-1 receptor-related kinase (IRAK- $)$ ). IRAK protein interacts and activates with TNF receptor-related factor 6 (TRAF6). TRAF6 recruits and activates TAK1. TAK1 activated nuclear factor $\kappa B$

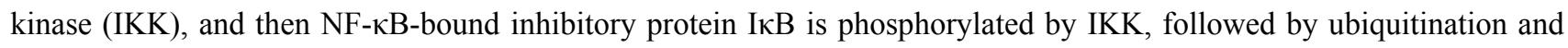
degradation, resulting in $\mathrm{p} 65-\mathrm{p} 50$, dimer of NF- $\mathrm{kB}$, loses to be inhibited, and then plays the role of its transcription factor and enters the nucleus to drive the transcription genes of inflammatory cytokines. ${ }^{34-36}$ Evidence has accumulated that the $\mathrm{NF}-\mathrm{\kappa B}$ signaling pathway is a pivotal pathway for macrophage activation and M1/M2 polarization. ${ }^{37-39}$ Our study indicated that CRAE caused the activation of TLR4, starting a series of signal cascade reactions, leading to NF- $\mathrm{KB}$ activation. The application of TLR4 and NF-KB inhibitor significantly inhibited the effect of CRAE-induced macrophage polarization, which highlighted the underlying mechanism.

Cimicifugae rhizome has been applied in traditional Chinese medicine clinical treatment for centuries. In the past, it was mainly used to treat wind heat headache, sore throat, uterine prolapse and so on. Since the 1990s, scholars have conducted a large number of in vitro activity screening studies on Cimicifugae rhizoma. C. dahurica is one of the three kinds of Cimicifugae rhizome. Experiments showed that the extract of $C$. dahurica has antitumor effects on human hepatoma cells (HepG2), human breast cancer cells (MCF-7, MDA-MB231), human glioma cells (SF-268), human leukemia cells (HL-60) and drug-resistant human hepatocellular carcinoma cell lines (R-HepG2), oral cancer. ${ }^{13,14,40}$ C. dahurica contains many active ingredients. The main anti-tumor components are considered triterpenoids. Interestingly, the results of this study showed that the components with polarization acting on macrophages in the microenvironment of myeloma may not be previously recognized triterpenoids. UHPLC-ESI- Q-Orbitrap-MS/MS was used to analyze the components of aqueous extract of the root of $C$. dahurica. According to mzCloud matching scores and TCMSP database, 10 compounds that may act on TLR4 were determined. According to results of computer 
molecular docking analysis, cimicifugic acid B had the highest binding energy with TLR4. It is worth noting that their interaction with TLR4 and the function mechanisms of these compounds need to be elucidated.

\section{Conclusion}

We provided the direct evidence for the polarization of aqueous extract of the root of Cimicifuga dahurica (CRAE) in myeloma-associated macrophages for the first time. We also illustrated that the CRAE activated TLR4-MyD88-TAK1$\mathrm{NF}-\kappa \mathrm{B}$ pathway to promote the macrophages differentiate into M1 macrophages, improved the tumor microenvironment, and inhibited tumor progression.

\section{Acknowledgments}

This work was supported by Jiangsu Graduate Training Innovation Project, Graduate Scientific Research and Practical Innovation Program (SJCX21_0782); Jiangsu Traditional Chinese Medicine Science and Technology Development Plan Project (ZD202003).

\section{Disclosure}

The authors report no conflicts of interest in this work.

\section{References}

1. Kyle RA, Rajkumar SV. Multiple myeloma. Blood. 2008;111(6):2962-2972. doi:10.1182/blood-2007-10-078022

2. Rajkumar SV. Treatment of multiple myeloma. Nat Rev Clin Oncol. 2011;8(8):479-491.

3. Anderson KC. The 39th David A. Karnofsky lecture: bench-to-bedside translation of targeted therapies in multiple myeloma. J Clin Oncol. 2012;30 (4):445-452. doi:10.1200/JCO.2011.37.8919

4. Mantovani A, Sozzani S, Locati M, Allavena P, Sica A. Macrophage polarization: tumor-associated macrophages as a paradigm for polarized M2 mononuclear phagocytes. Trends Immunol. 2002;23(11):549-555. doi:10.1016/S1471-4906(02)02302-5

5. Panchabhai S, Kelemen K, Ahmann G, Sebastian S, Mantei J, Fonseca R. Tumor-associated macrophages and extracellular matrix metalloproteinase inducer in prognosis of multiple myeloma. Leukemia. 2016;30(4):951-954. doi:10.1038/leu.2015.191

6. Andersen MN, Andersen NF, Rødgaard-Hansen S, Hokland M, Abildgaard N, Møller HJ. The novel biomarker of alternative macrophage activation, soluble mannose receptor (sMR/sCD206): implications in multiple myeloma. Leuk Res. 2015;39(9):971-975. doi:10.1016/j. leukres.2015.06.003

7. Zheng Y, Yang J, Qian J, et al. PSGL-1/selectin and ICAM-1/CD18 interactions are involved in macrophage-induced drug resistance in myeloma. Leukemia. 2013;27(3):702-710. doi:10.1038/leu.2012.272

8. Chen J, He D, Chen Q, et al. BAFF is involved in macrophage-induced bortezomib resistance in myeloma. Cell Death Dis. $2017 ; 8(11)$ :e3161. doi:10.1038/cddis.2017.533

9. Gutiérrez-González A, Martínez-Moreno M, Samaniego R, et al. Evaluation of the potential therapeutic benefits of macrophage reprogramming in multiple myeloma. Blood. 2016;128(18):2241-2252. doi:10.1182/blood-2016-01-695395

10. Wang Q, Lu Y, Li R, et al. Therapeutic effects of CSF1R-blocking antibodies in multiple myeloma. Leukemia. 2018;32(1):176-183. doi:10.1038/ leu. 2017.193

11. Ou T, Dai X, Sun X. Discussion on theory and practice of treating multiple myeloma based on shengma biejia decoction and beating chemotherapy. Chin J Tradit Chin Med. 2020;27:123-125. doi:10.19879/j.cnki.1005-5304.201901353

12. Dai H, Ma B, Dai X, et al. Shengma biejia decoction inhibits cell growth in multiple myeloma by Inducing autophagy-mediated apoptosis through the ERK/mTOR pathway. Front Pharmacol. 2021;12:585286. doi:10.3389/fphar.2021.585286

13. Guo Y, Yin T, Wang X, et al. Traditional uses, phytochemistry, pharmacology and toxicology of the genus Cimicifuga: a review. J Ethnopharmacol. 2017;209:264-282. doi:10.1016/j.jep.2017.07.040

14. Li JX, Yu ZY. Cimicifugae rhizoma: from origins, bioactive constituents to clinical outcomes. Curr Med Chem. 2006;13(24):2927-2951. doi:10.2174/092986706778521869

15. Trott O, Olson AJ. AutoDock Vina: improving the speed and accuracy of docking with a new scoring function, efficient optimization, and multithreading. J Comput Chem. 2010;31(2):455-461. doi:10.1002/jcc.21334

16. Zheng Y, Cai Z, Wang S, et al. Macrophages are an abundant component of myeloma microenvironment and protect myeloma cells from chemotherapy drug-induced apoptosis. Blood. 2009;114(17):3625-3628. doi:10.1182/blood-2009-05-220285

17. Giraldo NA, Becht E, Remark R, Damotte D, Sautès-Fridman C, Fridman WH. The immune contexture of primary and metastatic human tumours. Curr Opin Immunol. 2014;27:8-15. doi:10.1016/j.coi.2014.01.001

18. Fridman WH, Pagès F, Sautès-Fridman C, Galon J. The immune contexture in human tumours: impact on clinical outcome. Nat Rev Cancer. 2012;12(4):298-306. doi:10.1038/nrc3245

19. Murray PJ, Wynn TA. Protective and pathogenic functions of macrophage subsets. Nat Rev Immunol. 2011;11(11):723-737. doi:10.1038/nri3073

20. Murray PJ, Allen JE, Biswas SK, et al. Macrophage activation and polarization: nomenclature and experimental guidelines. Immunity. 2014;41 (1):14-20. doi:10.1016/j.immuni.2014.06.008

21. Chen X, Chen J, Zhang W, et al. Prognostic value of diametrically polarized tumor-associated macrophages in multiple myeloma. Oncotarget. 2017;8(68):112685-112696. doi:10.18632/oncotarget.22340 
22. Wang H, Hu WM, Xia ZJ, et al. High numbers of CD163+ tumor-associated macrophages correlate with poor prognosis in multiple myeloma patients receiving bortezomib-based regimens. J Cancer. 2019;10(14):3239-3245. doi:10.7150/jca.30102

23. Qian S, Zhang H, Dai H, et al. Is sCD163 a clinical significant prognostic value in cancers? A systematic review and meta-analysis. Front Oncol. 2020;10:585297. doi:10.3389/fonc.2020.585297

24. Saeedifar AM, Mosayebi G, Ghazavi A, Bushehri RH, Ganji A. Macrophage polarization by phytotherapy in the tumor microenvironment. Phytother Res. 2021;35(7):3632-3648. doi:10.1002/ptr.7058

25. Han X, Wei Q, Lv Y, et al. Ginseng-derived nanoparticles potentiate immune checkpoint antibody efficacy by reprogramming the cold tumor microenvironment. Mol Ther. 2021;30(1):327-340. doi:10.1016/j.ymthe.2021.08.028

26. Zhang Z, Fu X, Xu L, et al. Nanosized alumina particle and proteasome inhibitor bortezomib prevented inflammation and osteolysis induced by titanium particle via autophagy and NF-кB signaling. Sci Rep. 2020;10(1):5562. doi:10.1038/s41598-020-62254-X

27. Jiang L, Song J, Hu X, et al. The proteasome inhibitor bortezomib inhibits inflammatory response of periodontal ligament cells and ameliorates experimental periodontitis in rats. J Periodontol. 2017;88(5):473-483. doi:10.1902/jop.2016.160396

28. Meiners S, Ludwig A, Stangl V, Stangl K. Proteasome inhibitors: poisons and remedies. Med Res Rev. 2008;28(2):309-327. doi:10.1002/med.20111

29. Hu LH, Fan YJ, Li Q, et al. Bortezomib protects against dextran sulfate sodium-induced ulcerative colitis in mice. Mol Med Rep. 2017;15 (6):4093-4099. doi:10.3892/mmr.2017.6524

30. Moschetta M, Di Pietro G, Ria R, et al. Bortezomib and zoledronic acid on angiogenic and vasculogenic activities of bone marrow macrophages in patients with multiple myeloma. Eur J Cancer. 2010;46(2):420-429. doi:10.1016/j.ejca.2009.10.019

31. Kim J, Denu RA, Dollar BA, et al. Macrophages and mesenchymal stromal cells support survival and proliferation of multiple myeloma cells. $B r$ J Haematol. 2012;158(3):336-346. doi:10.1111/j.1365-2141.2012.09154.x

32. Li X, Liu R, Su X, et al. Harnessing tumor-associated macrophages as aids for cancer immunotherapy. Mol Cancer. 2019;18(1):177. doi:10.1186/ s12943-019-1102-3

33. Genin M, Clement F, Fattaccioli A, Raes M, Michiels C. M1 and M2 macrophages derived from THP-1 cells differentially modulate the response of cancer cells to etoposide. BMC Cancer. 2015;15:577. doi:10.1186/s12885-015-1546-9

34. Bryant CE, Symmons M, Gay NJ. Toll-like receptor signalling through macromolecular protein complexes. Mol Immunol. 2015;63(2):162-165. doi:10.1016/j.molimm.2014.06.033

35. Almog T, Kandel-Kfir M, Shaish A, et al. Knockdown of interleukin-1 $\alpha$ does not attenuate LPS-induced production of interleukin-1 $\beta$ in mouse macrophages. Cytokine. 2015;73(1):138-143. doi:10.1016/j.cyto.2015.01.029

36. Liu H, Chen K, Feng W, Wu X, Li H. TLR4-MyD88/Mal-NF-kB axis is involved in infection of HSV-2 in human cervical epithelial cells. PLoS One. 2013;8(11):e80327. doi:10.1371/journal.pone.0080327

37. Stout RD, Jiang C, Matta B, Tietzel I, Watkins SK, Suttles J. Macrophages sequentially change their functional phenotype in response to changes in microenvironmental influences. J Immunol. 2005;175(1):342-349. doi: 10.4049/jimmunol.175.1.342

38. Wang N, Liang H, Zen K. Molecular mechanisms that influence the macrophage m1-m2 polarization balance. Front Immunol. 2014;5:614. doi:10.3389/fimmu.2014.00614

39. Wanderley CW, Colón DF, Luiz JPM, et al. Paclitaxel reduces tumor growth by reprogramming tumor-associated macrophages to an M1 profile in a TLR4-dependent manner. Cancer Res. 2018;78(20):5891-5900. doi:10.1158/0008-5472.CAN-17-3480

40. Wu L, Chen ZL, Su Y, Wang QH, Kuang HX. Cycloartenol triterpenoid saponins from cimicifuga simplex (Ranunculaceae) and their biological effects. Chin J Nat Med. 2015;13(2):81-89. doi:10.1016/S1875-5364(15)60011-5

Journal of Inflammation Research

\section{Dovepress}

\section{Publish your work in this journal}

The Journal of Inflammation Research is an international, peer-reviewed open-access journal that welcomes laboratory and clinical findings on the molecular basis, cell biology and pharmacology of inflammation including original research, reviews, symposium reports, hypothesis formation and commentaries on: acute/chronic inflammation; mediators of inflammation; cellular processes; molecular mechanisms; pharmacology and novel anti-inflammatory drugs; clinical conditions involving inflammation. The manuscript management system is completely online and includes a very quick and fair peer-review system. Visit http://www.dovepress.com/testimonials.php to read real quotes from published authors.

Submit your manuscript here: https://www.dovepress.com/journal-of-inflammation-research-journal 